\title{
Multiferroic Materials: Physics and Properties
}

AJC Buurma, GR Blake, and TTM Palstra, University of Groningen, Groningen, The Netherlands

U Adem, İzmir Institute of Technology, Urla, İzmir, Turkey

(C) 2016 Elsevier Inc. All rights reserved.

\begin{tabular}{|c|c|c|}
\hline 1 & Introduction & 1 \\
\hline 2 & Importance and Applications & 2 \\
\hline 3 & Historical Perspective & 4 \\
\hline 4 & Microscopic Origin & 4 \\
\hline 4.1 & Magnetism & 4 \\
\hline 4.2 & Ferroelectrics & 4 \\
\hline 5 & Types of Multiferroic Materials & 5 \\
\hline 5.1 & Type I Multiferroics & 5 \\
\hline 5.1 .1 & $\mathrm{ABO}_{3}$ perovskites & 5 \\
\hline 5.1.1.1 & $\mathrm{BiFeO}_{3}$ & 5 \\
\hline 5.1.1.2 & $\mathrm{EuTiO}_{3}$ & 6 \\
\hline 5.1 .2 & Geometric ferroelectrics & 6 \\
\hline 5.1.2.1 & $\mathrm{AMnO}_{3}$ hexagonal manganites & 6 \\
\hline 5.1.2.2 & Hexagonal $\mathrm{RFeO}_{3}(\mathrm{R}=\mathrm{Y}$, Dy-Lu) & 7 \\
\hline 5.1.2.3 & $\mathrm{BaMF}_{4}$ & 7 \\
\hline 5.1 .3 & $\mathrm{M}_{3} \mathrm{~B}_{7} \mathrm{O}_{13} \mathrm{X}$ boracites & 7 \\
\hline 5.1 .4 & Organic-inorganic hybrids & 8 \\
\hline 5.1 .5 & Octahedral rotations and hybrid improper ferroelectricity & 8 \\
\hline 5.1 .6 & Charge order & 8 \\
\hline 5.1.6.1 & Half-doped manganites & 8 \\
\hline 5.1.6.2 & $\mathrm{LuFe}_{2} \mathrm{O}_{4}$ & 8 \\
\hline 5.2 & Type II Multiferroics: Improper Ferroelectricity Induced by Spin Order & 9 \\
\hline 5.2 .1 & Cycloidal spin spirals $\left(\mathrm{RMnO}_{3}\right)$ & 9 \\
\hline 5.2 .2 & p-d Hybridization & 10 \\
\hline 5.2 .3 & Proper screw spin order & 11 \\
\hline 5.2 .4 & Ferroaxial multiferroics $\left(\mathrm{CaMn}_{7} \mathrm{O}_{12}\right)$ & 11 \\
\hline 5.2 .5 & Symmetric exchange striction & 11 \\
\hline 5.2.5.1 & $\mathrm{Ca}_{3} \mathrm{CO}_{2}-\mathrm{x} \mathrm{Mn}_{\mathrm{x}} \mathrm{O}_{6}$ & 11 \\
\hline 5.2.5.2 & $\mathrm{RMn}_{2} \mathrm{O}_{5}$ & 12 \\
\hline 5.2.5.3 & E-type $\mathrm{RMnO}_{3}$ & 12 \\
\hline 6 & Single Phase Multiferroic Thin Films & 12 \\
\hline 7 & Composite Structures & 13 \\
\hline 8 & Interfacial Magnetoelectric Coupling with Electronic Effects in Multiferroic Heterostructures & 14 \\
\hline 9 & Multiferroic Domain Walls and Emergent Functional Properties at the Domain Walls & 14 \\
\hline Refere & & 14 \\
\hline
\end{tabular}

\footnotetext{
Change History: August 2015. T.T.M. Palstra. The field of multiferroics has been very active and a considerable expansion of the original chapter was necessary. The following additions and important changes have been made to the previous version, minor changes are omitted in order to avoid prolixity. Thin film memory devices are discussed in paragraph 2 as a potential application of multiferroics. Device geometry of the relevant devices is shown in Figure 2. Paragraph 5 and subsections have been changed considerably. It is now common practice in the field to attribute multiferroic materials to two distinct classes. Multiferroics are coined either type I (5.1 and subsections) or type II (5.2 and subsections), depending on whether the magnetic and ferroelectric order parameters arise as independently, or show a coupling interaction. Sections 5.1.2 to 5.1.6: quantum paraelectric $\mathrm{EuTiO}_{3}, \mathrm{hexagonal}_{\mathrm{RFeO}}$ and $\mathrm{M}_{3} \mathrm{~B}_{7} \mathrm{O}_{13} \mathrm{X}$ boracites, Organic-Inorganic hybrids, charge ordered multiferroics and hybrid improper ferroelectrics have been added as class I multiferroics. The paragraph on Hexagonal $\mathrm{AMnO}_{3}$ manganites has been updated. For class II multiferroics, the important magnetoelectric coupling mechanism is reviewed. Each coupling mechanism is discussed in newly written paragraphs. The magnetoelectric coupling mechanism in spin spirals $\left(\mathrm{RMnO}_{3}\right)$ is explained in Section 5.2.1. Newly added Figure 5 presents a graphical overview of the relevant magnetoelectric coupling phenomena in type II multiferroics. These newly discovered magnetoelectric cross-coupling mechanisms are now described in Sections 5.2.2 to 5.2.5. Figures 6 and 7 have been added to represent the $\mathrm{RMn}_{2} \mathrm{O}_{5}$ and E-type ordered $\mathrm{RMnO}_{3}$ structures. Sections $6,7,8$ review recent developments in thin film multiferroic heterostructures. The relevant interfacial magnetoelectric coupling mechanisms are discussed. Finally, multiferroic domain walls are briefly discussed.
} 


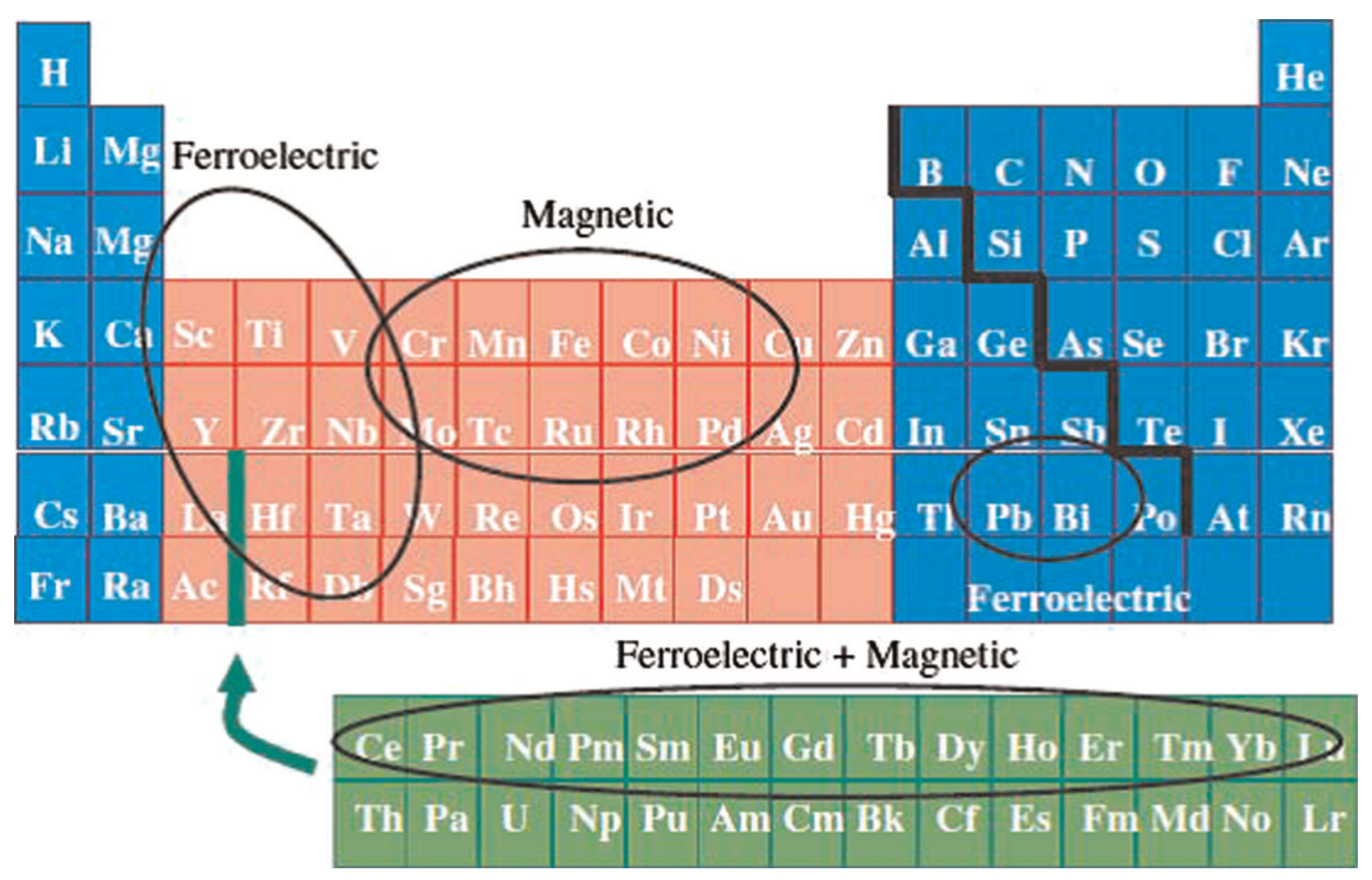

Figure 1 Periodic table of the elements highlighting the elements generating magnetic and ferroelectric behavior.

\section{Introduction}

Multiferroics are materials that simultaneously exhibit more than one type of ordering, including magnetic, electric, and elastic order. Magnetic order is conventionally driven by exchange interactions between magnetic dipoles, originating from unfilled shells of electron orbitals. Electric order is the result of ordering of local electric dipoles. Elastic order is the result of ordering of atomic displacements due to strain. The simultaneous occurrence of magnetic and electric order is particularly interesting as it combines properties that could be utilized for information storage, processing, and transmission. It allows both magnetic and electric fields to interact with magnetic and electric order. However, this property is exceedingly rare as the existence of partially filled atomic orbitals, prerequisite for magnetic dipoles or moments, usually precludes the occurrence of local electric dipoles, which are typically associated with the presence of either empty d-shells and/or an electron lone-pair configuration (see Figure 1). For practical purposes, we will limit the discussion to the simultaneous ordering of magnetic and electric dipoles in uniform compounds (excluding composite materials), the so-called multiferroics. This class of materials exhibits ferroelectric ordering combined with ferro- or antiferromagnetic ordering. Ferro-ordering occurs when all dipoles are oriented in the same direction, whereas antiferro-ordering involves a ferro-ordering of two or more sublattices in opposite directions.

Here, an overview of multiferroic materials is given, with the emphasis on covering the different classes of multiferroic materials, physical origins of the mechanisms that can be used to distinguish between these different classes and information about the physical properties of these materials related to multiferroicity, such as the crystal structure, magnetic and ferroelectric properties.

There are quite a few review articles on multiferroic materials to which we refer the reader for further information. ${ }^{1-5}$ In this review, we provide the reader with an up-to-date text, covering a wide range of multiferroic materials, including the recent developments in the field. We do not intend to provide an exhaustive list of all multiferroics. Rather, we focus on the specific materials that epitomize a particular manifestation of magnetoelectric coupling.

\section{Importance and Applications}

Ferromagnetic materials are commonly used for non-volatile information storage in tapes, hard drives, etc. They are also used for information-processing due to the interaction of electric current and light with magnetic order. Ferroelectrics find applications due to their large piezoelectric-coupling constant, that is, the coupling between an electric field and strain. This effect is utilized in 
devices such as capacitors, microphones, and transducers where a voltage can generate strain, and vice versa. Ferroelectric materials not only exhibit piezoelectric coupling, common for most materials that lack inversion symmetry, but also possess memory functionality. The electric polarization remains finite after removing an applied electric held. This property can be exploited in nonvolatile memory devices, where the information stored in the electric polarization is retained, even after removing the power of the device. Such devices are currently being introduced in the market. Much interest is being generated by multiferroics. The simultaneous magnetic and electric order makes it possible for the magnetic polarization to be addressed or switched not only by applying a magnetic field but also by an electric field, or likewise the electric polarization can be addressed or switched by applying an electric and/or a magnetic field. This property enables completely new device architectures to be designed. Furthermore, it aids materials scientists to understand the associated chemical bonding that will allow them to circumvent the prevalent mutual exclusion of magnetic and electric dipoles by smart materials engineering in bulk compounds in thin films.

The most promising application of multiferroics is a multiferroic memory device where information can be written electrically taking the advantage of lower power operation and read magnetically thus non-destructively. ${ }^{6}$ To achieve this in microelectronic devices, the materials need to be highly insulating, have large and switchable polarization and large coupling between electric and magnetic order at room temperature. Most multiferroics do not satisfy these conditions as we explain in the following sections.

There is, however, promising progress toward applications. We refer to a review article by J.F. Scott for the applications of multiferroics. ${ }^{7}$ Multiferroic materials are being tested as different elements in spintronics such as spin valves, magnetic tunnel junctions or spin filters. ${ }^{7,8} \mathrm{BiFeO}_{3}$ is mainly used as the multiferroic material in these devices.

Heterostructures of ferromagnetic and multiferroic thin-film layers are used in multiferroic device prototypes. The electronic magnetoelectric coupling mechanisms mediated by interfacial effects (see Section 8) in these heterostructures enable practical devices for memory applications. The multiferroic tunnel junction (MFTJ) device, ${ }^{9-11}$ as shown in Figure 2(a), can be used as a four-state ferroelectric spin-valve. MFTJs combine the Tunnel Electro Resistance (TER) effect, in which the tunnel barrier height depends on the polarization state of the ferroelectric ${ }^{12}$ and the tunnel magnetoresistance (TMR) effect in which the tunneling transport depends on the magnetization orientation of top and bottom electrodes. ${ }^{13}$ For a review on multiferroic devices we refer the reader to Tsymbal et al. ${ }^{14}$

Nonvolatile magnetic random access (MRAM) memories employ a magnetic tunnel junction that consists of a tunnel barrier that is sandwiched between free ferromagnetic top and magnetically pinned bottom electrodes. ${ }^{8}$ MRAM offers high access speed, but currently requires a spin polarized current to reverse the magnetization of the free FM layer using spin transfer torque. ${ }^{15}$ A writing process that is based on induced currents imposes limits on miniaturization of the access transistors and causes energy dissipation. The manipulation of the magnetization by an electric field is therefore a property that is much sought after in the design of a MERAM memory element. Figure 2(b) shows such a memory cell that employs an electric field controlled write instead of a conventional dissipative electric current induced write. MERAM can offer improved storage density against reduced energy consumption.

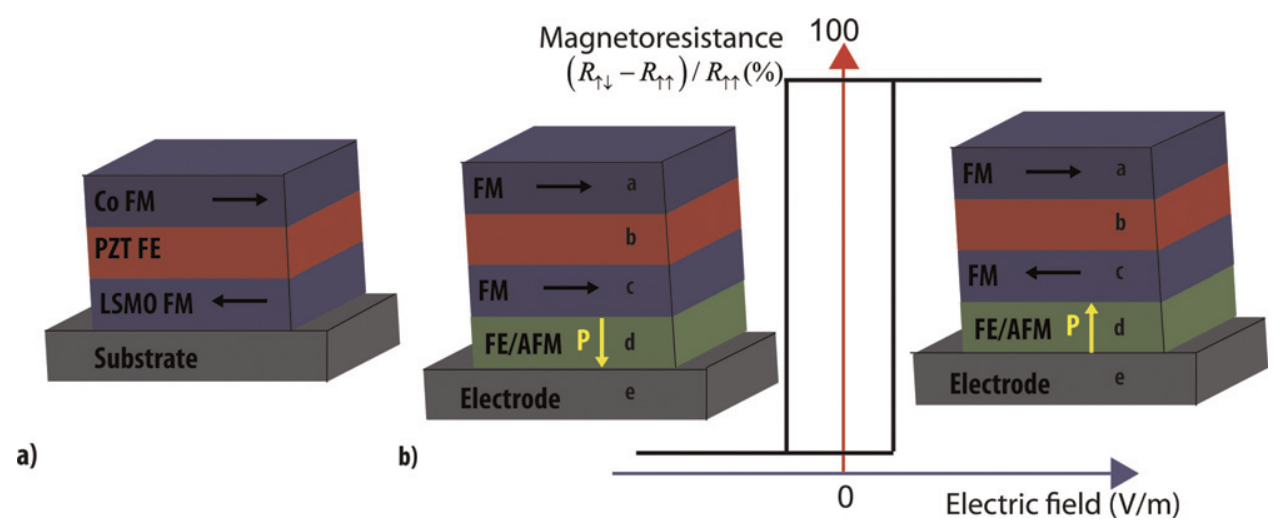

Figure 2 (a) Multiferroic tunnel junction with a top ferromagnetic cobalt layer and a bottom ferromagnetic $\mathrm{La}_{0.7} \mathrm{Sr}_{0.3} \mathrm{MnO}_{3}$ ( $\mathrm{LSMO}$ layer shown in blue, separated by an ultrathin ferroelectric PZT $\left(\mathrm{PbZr}_{0.2} \mathrm{Ti}_{0.8} \mathrm{O}_{3}\right)$ tunnel barrier shown in red. The combined Tunneling Electro Resistance (TER) and Tunnel Magneto Resistance (TMR) effects enable switching between four resistance states. Device structure reproduced from Quindeau, A., et al., 2015. Four-state ferroelectric spin-valve. Scientific Reports 5, 09749. (b) Proposed Magneto-Electric Random Access Memory (MERAM) device geometry, reproduced from Bibes, M., Barthelemy, A., 2008. Multiferroics: Towards a magnetoelectric memory. Nature Materials 7 (6), $425-426$. Magnetoelectric coupling at the FE/FM interface (layers $\mathrm{c}$ and $\mathrm{d}$ respectively) enables switching between two resistive states in response to an electric field. This electric field is applied at layers $c$ and e and switches the electric polarization (shown as a yellow arrow) of an antiferromagnetic and ferroelectric layer $d$ (shown in green) with a concomitant reversal of the magnetization of coupled FM layer c. (Left) Low resistive state in which the parallel orientation of the magnetizations in layers a and $\mathrm{c}$ allow tunneling across the magnetic tunnel junction. (Right) Antiparallel magnetization configuration of the top and bottom ferromagnetic layer leading to a highly resistive state. 


\section{Historical Perspective}

The magnetoelectric effect - the induction of magnetization by an electric field and the induction of electric polarization by a magnetic field - dates back to Pierre Curie, who argued that it was possible for such coupling to exist. However, it was later argued on the basis of symmetry considerations that no such effect could occur. After the discovery and exploitation of the properties of ferroelectric materials such as $\mathrm{BaTiO}_{3}$ in the 1940s, the study of multiferroic materials gained prominent interest in the 1960s. In 1982, a review article on multiferroics by Smolenskii and Chupis listed some 50 multiferroic materials. ${ }^{1}$

In the last few years, additional mechanisms giving rise to ferroelectricity have been identified, both experimentally and theoretically, and have resulted in novel multiferroics. This development has been largely driven by the discovery of ferroelectricity in $\mathrm{TbMnO}_{3}$ by Kimura et al. ${ }^{16}$ Here, the electric order is associated with an incommensurate spin order of the manganese ions. Detailed mechanisms of spin-induced polarization will be discussed in Section 5.2. Furthermore, progress in the theoretical understanding of the chemical bonding associated with ferroelectricity has impacted greatly on the understanding of multiferroics. $^{17}$

\section{Microscopic Origin}

\subsection{Magnetism}

The occurrence of magnetic order is based on the presence of magnetic moments and their interactions. At room temperature, magnetic order in solids is mostly restricted to partially filled d- or f-shells. The f-shell elements include the rare-earths and the actinides. As the f-electrons are shielded by the valence electrons from neighboring atoms, their interactions are typically weak, and ordering is mostly restricted to temperatures below $50 \mathrm{~K}$. Magnetism for the d-shell materials, the transition metals, is mostly restricted to the $3 \mathrm{~d}$-elements, as the $4 \mathrm{~d}$ - and $5 \mathrm{~d}$-elements often exhibit a low spin-state. The largest magnetic moments can be found near the middle of the series, including $\mathrm{Mn}, \mathrm{Fe}, \mathrm{Ni}$, and Co. Here, strong direct exchange interactions can lead to magnetic order far above room temperature. However, this typically leads to metallic behavior, precluding electric order, because any local electric dipole will be fully screened. Therefore, multiferroic behavior is restricted to materials with indirect exchange interactions such as oxides, where the magnetic interactions are mediated by the ligands of the transition metal. Although this reduces the magnetic ordering temperatures, many oxides nonetheless exhibit magnetic order at above room temperature. Ferromagnetic interactions in oxides are usually driven by the double-exchange interaction. The resulting metallic state screens electric dipole formation, as is the case for direct interactions. Therefore, ferromagnetic interactions for multiferroics should originate from $90^{\circ}$ superexchange or from spin canting of antiferromagnets, induced by local anisotropy effects or Dzyaloshinskii-Moriya coupling.

\subsection{Ferroelectrics}

The origin of ferroelectricity is commonly associated with the occurrence of a soft mode or a polarization catastrophe. ${ }^{18}$ One can easily show that a cation, represented by a point charge, is never stable in the center of its anion ligands. The position of a cationic transition metal with respect to its ligands is the result of a balance between the attractive Coulomb force of the ions and the electronic repulsion between the core electrons. Conventionally, ionic crystal structures are derived from a dense packing of anions, such as the face-centered-cubic oxygen sub-lattice in the spinels. The cations then fill the interstitial sites. Such dense packing usually leads to centered structures. Off-center positions are restricted to small ionic radius ions such as lithium, and are usually not observed for transition metals with a partially filled d-shell, necessary for magnetism. The first group of ferroelectrics is thus found for the $\mathrm{d}^{0}$-elements such as $\mathrm{Ti}^{4+}, \mathrm{Zr}^{4+}$, and $\mathrm{Nb}^{5+}$. These oxides are known as titanates, zirconates, and niobates. A second group of ferroelectrics is found for materials that have a lone-pair configuration, often found in heavy p-elements such as $\mathrm{Pb}^{2+}$ and $\mathrm{Bi}^{3+}$. Here, the interaction of the lone pair with the ligands results in an off-center position for the cation. The largest polarization is observed for materials in which both mechanisms operate simultaneously, such as $\mathrm{Pb}(\mathrm{Zr}, \mathrm{Ti}) \mathrm{O}_{3}(\mathrm{PZT})$, in which $\mathrm{Pb}^{2+}$ and the $\mathrm{d}^{0} \mathrm{Zr}^{4+}$ - and $\mathrm{Ti}^{4+}$-ions contribute to the ferroelectric polarization. The requirement for ferroelectrics to reverse their polarization on applying an electric field necessitates a total energy landscape containing multiple accessible minima with finite polarization.

Before proceeding further, a remark on precise terminology is in order.

- Ferroelectrics are materials with a spontaneous electric polarization below an ordering temperature. They lack inversion symmetry and an improper rotation axis. Most importantly, the polarization can be switched by an applied electric field. The switching of the polarization is associated with the reversal of the polarization direction of domains by rotation and/or domain growth. The orientation of domains in ferroelectrics is analogous to the situation for the polarization of ferromagnets.

- Pyroelectrics are a more general class of materials that exhibit a spontaneous polarization below an ordering temperature. Ferroelectrics are a subgroup of pyroelectrics. However, in pyroelectrics the electric field required for polarization reversal may exceed the electric-breakdown field, originating from catastrophic impact ionization. This means that the polarization may not 
be reversible by applying electric fields. The polarization may be switched by cooling in an applied field through the ordering transition. Cooling in zero-field can result in twinned samples, for example, inversion twins.

- Proper ferroelectrics are materials in which the primary mode responsible for the phase transition also causes the ferroelectric ordering. The responsible mode can be traced from the change in symmetry through the transition. The polarization is typically larger than $1 \mu \mathrm{C} \mathrm{cm}^{-2}$.

- Improper ferroelectrics are materials in which the ferroelectric order is driven not by the primary order parameter, but a secondary order parameter. A typical example is the ferroelectric ordering in $\mathrm{TbMnO}_{3}$, in which the electric order is a by-product of the magnetic ordering. The nature of the transition follows from group theoretical analysis. The polarization is typically smaller than $1 \mu \mathrm{C} \mathrm{cm} \mathrm{cm}^{-2}$.

- Antiferroelectrics are materials with local electric dipoles arranged in antiparallel fashion. An example is $\mathrm{PbZrO}_{3}$, which exhibits antiferroelectric hysteresis and saturation in applied electric fields. Antiferroelectrics are members of the more general class of antipolar materials, which have antiparallel electric ordering but cannot be polarized. ${ }^{19}$

\section{Types of Multiferroic Materials}

We distinguish two main classes of multiferroics. ${ }^{2}$ The first class, Type I multiferroics, comprises materials in which the ferroic orders arise independently of each other. These materials can show high polarization values and high ordering temperatures of the individual order parameters. However, the mutual interactions between the magnetic and ferroelectric orders are typically very weak.

The second class, Type II multiferroic materials, shows ferroelectric polarization as a by-product of another driving order parameter, such as spin order.

\subsection{Type I Multiferroics}

\subsection{1 $\mathrm{ABO}_{3}$ perovskites}

This structure class is the same as that of many ferroelectric materials such as $\mathrm{BaTiO}_{3}$, where $\mathrm{BO}_{6}$ octahedra share corners and the A-ion is coordinated by eight octahedra (see Figure 3). The ferroelectric moment can, in part, be derived from a lone-pair ion on the A-site, such as $\mathrm{Pb}^{2+}$ or $\mathrm{Bi}^{3+}$, and in part from a small transition-metal ion, with $\mathrm{d}^{0}$ electron configuration, on the B-site. Magnetism can be generated by a transition-metal ion on the B-site with a partially filled d-electron shell, such as $\mathrm{Mn}^{3+} \mathrm{or} \mathrm{Fe}^{3+}$, or by a rare-earth ion on the A-site. A judicious choice of composition can lead to multiferroicity in compounds such as $\mathrm{BiFeO}_{3}{ }^{20}$ $\mathrm{Pb}\left(\mathrm{Fe}_{2 / 3} \mathrm{~W}_{1 / 3}\right) \mathrm{O}_{3}{ }^{21}$ and various other combinations (see Figure 2).

\subsubsection{1 $\mathrm{BiFeO}_{3}$}

Being one of the very few room temperature multiferroics, $\mathrm{BiFeO}_{3}$ is arguably the most important multiferroic. Ferroelectric order originates from the lone electron pair of $\mathrm{Bi}^{3+}$, while magnetic ordering is due to the unpaired Fe spins. The ferroelectric transition temperature is $1143 \mathrm{~K}$ while antiferromagnetic ordering sets in below $643 \mathrm{~K}$. The antiferromagnetic ordering is complicated by canting of the spins and the formation of a long-range spin cycloid that is incommensurate with the lattice. ${ }^{20} \mathrm{~A}$ surge in research on $\mathrm{BiFeO}_{3}$ started after the report of large ferroelectric polarization $\left(60 \mu \mathrm{C} \mathrm{cm}^{-2}\right)$ in thin films of $\mathrm{BiFeO}_{3}{ }^{22}$ Prior to this report, ferroelectric measurements on bulk $\mathrm{BiFeO}_{3}$ did not give such large polarization values due to the high conductivity of the samples caused by oxygen vacancies. It was later shown that charged defects like oxygen vacancies impede the motion of domain walls, causing non-saturated ferroelectric hysteresis loops. ${ }^{23}$ Quenching from above the ferroelectric transition temperature causes
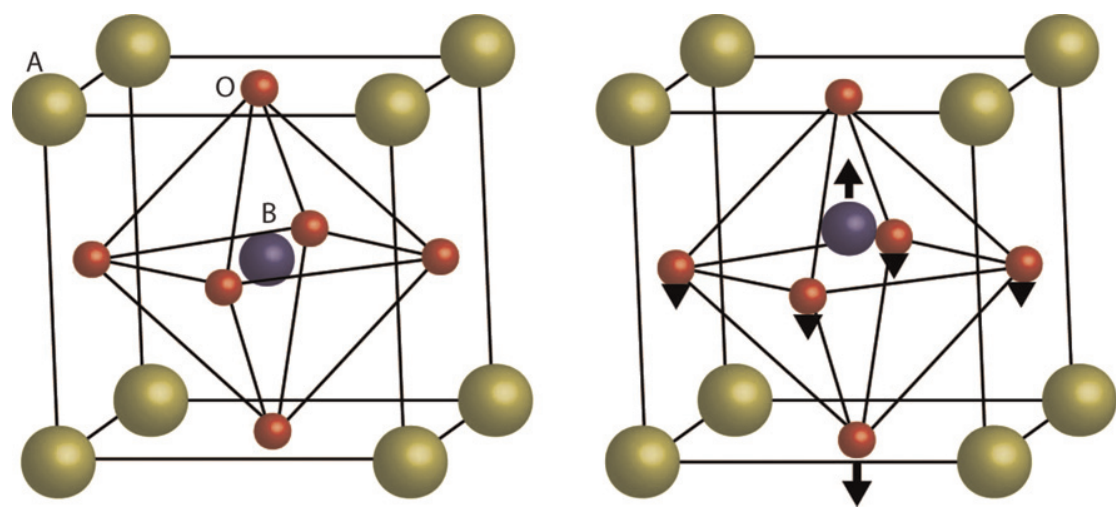

Figure 3 Crystal structure of a perovskite $\mathrm{ABO}_{3}$ with a tetragonal ferroelectric distortion. 
randomization of the defects and yields saturated loops with respectable polarization values $\left(\sim 20 \mu \mathrm{C} \mathrm{cm}{ }^{-2}\right)$, but still not comparable to those achieved in thin films and single crystals. Insulating single crystals of $\mathrm{BiFeO}_{3}$ were eventually grown after the report on thin films, and measurements on these samples confirmed the magnitude of electrical polarization in thin films. ${ }^{24}$ Since the ferroelectricity and magnetism originate independently of each other, magnetoelectric coupling is expected to be low. However, recently it has been shown that switching of the direction of magnetization is possible in two steps by applying an electric field to $\mathrm{BiFeO}_{3}$ thin films. This switching has also been exploited in a spin-valve device operating at room temperature. ${ }^{25}$

\subsubsection{2 $\mathrm{EuTiO}_{3}$}

$\mathrm{EuTiO}_{3}$ belongs to a class of materials called 'quantum paraelectrics' which are materials in which an incipient transition into a ferroelectric phase is suppressed close to $0 \mathrm{~K}$, where 'quantum fluctuations' become effective as the thermal vibrations cease. Eu ${ }^{2+}$ contains localized $4 \mathrm{f}$ spins which order antiferromagnetically at $5.5 \mathrm{~K}$. Below this temperature the dielectric constant strongly decreases, resulting in large coupling effects which are explained by the coupling of the spins to the optical phonon modes involved in the incipient ferroelectric transition. ${ }^{26}$ Application of a magnetic field suppresses magnetic ordering of the Eu spins, yielding large changes in the dielectric constant $(7 \%$ at $1.5 \mathrm{~T})$. Despite the need for low temperatures, quantum paraelectric materials with magnetic ordering constitute a promising class for achieving large coupling effects. Quantum paraelectrics can also be forced into a ferroelectric phase, for example, by using mismatch strain imposed on the thin film by the substrate. This has been realized in thin films of $\mathrm{EuTiO}_{3}$ grown on $\mathrm{DyScO}_{3}$ substrates. Using the mismatch strain, $\mathrm{EuTiO}_{3}$ becomes ferroelectric below $250 \mathrm{~K}$. Additionally, strain also tunes the magnetic order from an antiferromagnetic to a ferromagnetic ground state with a Curie temperature of $4.2 \mathrm{~K}^{27}$

\subsubsection{Geometric ferroelectrics}

\subsubsection{1 $\mathrm{AMnO}_{3}$ hexagonal manganites}

Despite the compositional identity with the perovskites, these materials are structurally completely different. They consist of sheets of $\mathrm{MnO}_{5}$ triangular bipyramids connected by $\mathrm{AO}_{7}$ polyhedra (see Figure 4). The magnetic ordering is derived from the electronic $\mathrm{d}^{4}$ configuration of $\mathrm{Mn}^{3+}$ on the B-site, or from a magnetic rare-earth ion on the A-site. Despite the contrary conclusions of early structure determinations, the $\mathrm{Mn}^{3+}$ is located close to the barycenter of the bipyramid, ${ }^{28}$ and the ferroelectric moment is largely derived from shifts of the $\mathrm{A}^{3+}$ in its coordination sphere. The structure is only stable for small rare-earth ions on the A-site,

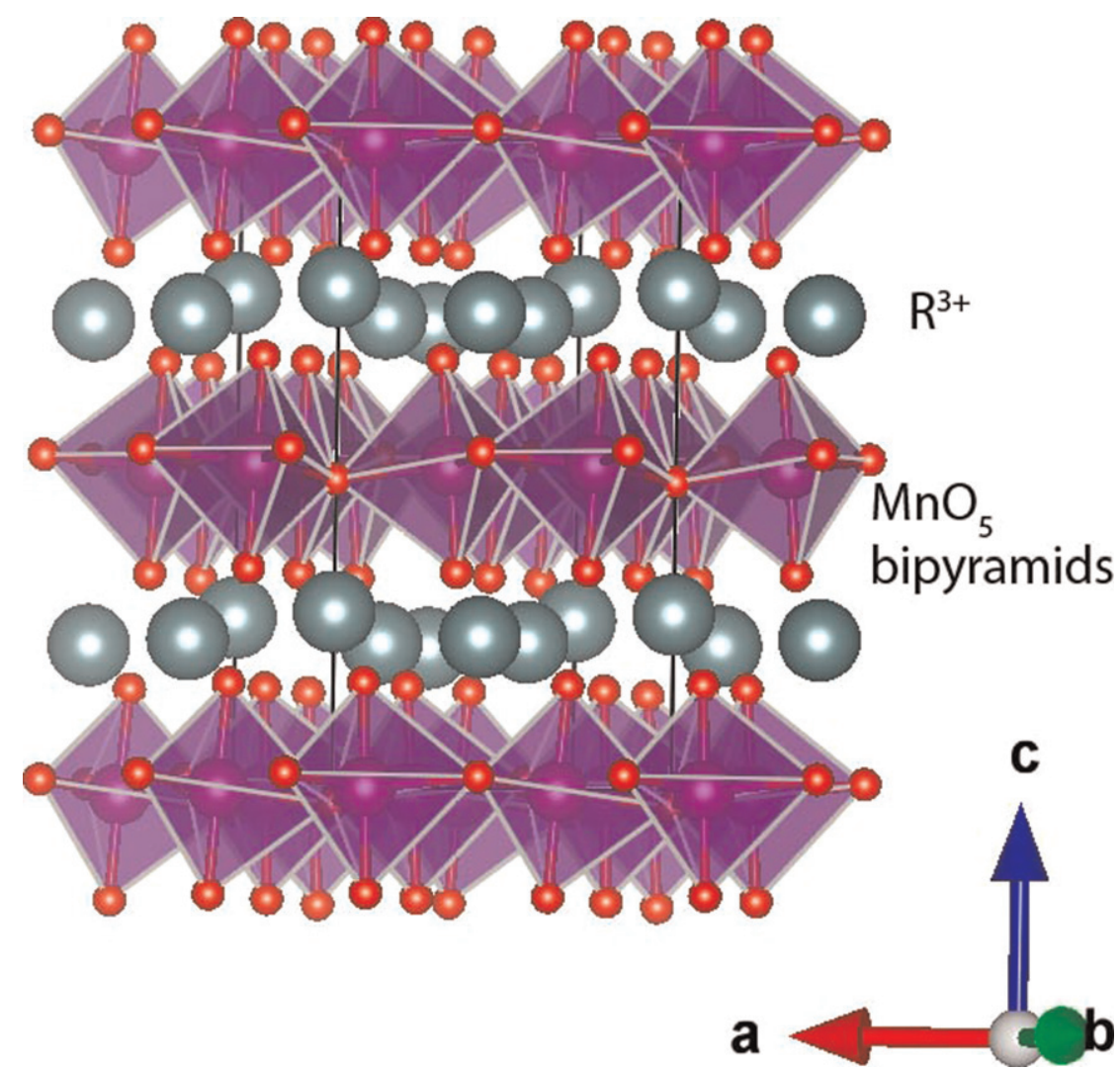

Figure 4 Crystal structure of a hexagonal manganite $\mathrm{RMnO}_{3}$, with $\mathrm{R}$ a rare-earth element, with ferroelectric distortion. 
including yttrium, and for $\mathrm{Mn}^{3+}$ on the B-site. The $\mathrm{Mn}^{3+}$ ion stabilizes the structure by a ligand-field effect, because the crystalfield splitting of the configuration in a triangular bipyramid results in a non-degenerate electronic state. For all other transition metals on the B-site and large ionic radius elements on the A-site, the perovskite structure is preferred. ${ }^{29,30}$

These materials were intensely studied in the 1960s, during which time the crystal and magnetic structure were investigated. ${ }^{31}$ The ferroelectric ordering sets in above $1000 \mathrm{~K}$ while antiferromagnetic ordering of the Mn-spins occurs near $75 \mathrm{~K}$, with small variations for different $\mathrm{R}$. These ordering temperatures indicate that $\mathrm{RMnO}_{3}$ are proper ferroelectrics, which is in line with a polarization of $5 \mu \mathrm{C} \mathrm{cm}^{-2}$ and large displacements inside the unit cell due to the electric ordering. Various corresponding indates $\mathrm{RInO}_{3}$ and gallates $\mathrm{RGaO}_{3}$ are also reported or predicted to be ferroelectric (see review by Abrahams ${ }^{29}$ ). Magnetoelectric coupling has been demonstrated by anomalies in the temperature dependence of the dielectric constant at the magnetic ordering temperature. ${ }^{32,33}$ The coupling was convincingly shown by Fiebig et al. ${ }^{34}$ in non-linear optical experiments, which mapped both electric and antiferromagnetic domain walls. Furthermore, Lottermoser et al. ${ }^{35}$ demonstrated that in $\mathrm{HoMnO}_{3}$, ferromagnetic ordering of the Ho-spins could reversibly be switched on and off by using an electric field, again indicating magnetoelectric coupling. There have been conflicting results from studies of the nature of the ferroelectric ordering. After the discovery of ferroelectric properties of this class of materials by Bertaut et al., ${ }^{31}$ several transition temperatures were reported. Measurements of the pyroelectric current of $\mathrm{YMnO}_{3}$ by Izmailzade and Kizhaev (1965) showed the ferroelectric ordering to be near $930 \mathrm{~K}{ }^{36} \mathrm{~A}$ transition at $1270 \mathrm{~K}$ in $\mathrm{YMnO}_{3}$ was reported by Lukaszewicz and Karut-Kalicinska ${ }^{37}$ with a tripling of the lattice. Van Aken $e t$ al. ${ }^{28}$ redetermined the crystal structures of several $\mathrm{RMnO}_{3}$ and found, in contrast with earlier reports, that $\mathrm{Mn}^{3+}$ is close to the barycenter of the coordinating oxygen bipyramid. They concluded that the ferroelectric moment is mostly associated with the relative motion of the R-ion and its coordinating oxygens.

Subsequent neutron diffraction experiments up to $1400 \mathrm{~K}$ by Lonkai et al. ${ }^{38}$ did not reveal any significant coupling to the modes responsible for the ferroelectric transition. They concluded that $\mathrm{RMnO}_{3}$ must be an improper ferroelectric, in agreement with a theoretical analysis of the displacements. ${ }^{39}$ Nénert et al. ${ }^{40}$ argued, based on synchrotron single-crystal X-ray diffraction and group theoretical analysis, that these materials are proper ferroelectrics, in agreement with the result of Izmailzade and Kizhaev. $^{36}$

Further analysis by Gibbs et al. in a comprehensive neutron diffraction study has shown that the polarization is driven by a non-polar tilting mode $\mathrm{K}_{3}$ as the primary order parameter, confirming the improper ferrielectric nature of $\mathrm{YMnO}_{3}$. They observed a structural unit cell tripling transition at $1258 \mathrm{~K}$, followed by an additional isosymmetric transition at $920 \mathrm{~K}$, with a concomitant decrease in polarization and the absence of any distinct intermediate phase. ${ }^{41}$

\subsubsection{Hexagonal $\mathrm{RFeO}_{3}(\mathrm{R}=\mathrm{Y}, \mathrm{Dy}$-Lu)}

Despite the similarity of the ionic radii of $\mathrm{Mn}^{3+}$ and $\mathrm{Fe}^{3+}, \mathrm{RFeO}_{3}$ is stable in the orthorhombic perovskite crystal structure under ambient conditions, unlike $\mathrm{RMnO}_{3}$. However, $\mathrm{RFeO}_{3}$ can also be stabilized in hexagonal symmetry using different synthesis techniques including epitaxial thin film growth. For example, thin films of $\mathrm{LuFeO}_{3}$ grown by Pulsed Laser Deposition (PLD) on $\alpha-\mathrm{Al}_{2} \mathrm{O}_{3}(001)$ substrates have hexagonal symmetry with the same space group as hexagonal manganites at room temperature, $\mathrm{P}_{3} \mathrm{~cm}^{42}$ Thanks to the larger moment of $\mathrm{Fe}^{3+}$ in the same triangular arrangement of the a-b plane of the hexagonal lattice, h$\mathrm{RFeO}_{3}$ is expected to have higher magnetic ordering temperatures than h- $\mathrm{RMnO}_{3}$. Indeed, for $\mathrm{LuFeO}_{3}$ thin films, antiferromagnetic ordering sets in below $440 \mathrm{~K}$ and below $130 \mathrm{~K}$ a spin reorientation transition introduces weak ferromagnetism. Ferroelectric switching has been demonstrated using piezoelectric force microscopy measurements at room temperature. Ferroelectric order persists up to $1050 \mathrm{~K}$ in $\mathrm{LuFeO}_{3}$, rendering it a room temperature multiferroic. ${ }^{43}$

\subsubsection{3 $\mathrm{BaMF}_{4}$}

This is an orthorhombic class of compounds where $\mathrm{M}$ is $\mathrm{Mn}, \mathrm{Fe}, \mathrm{Co}$, or Ni. The lattice consists of layers of vertex-sharing $\mathrm{MF}_{6}$ octahedra separated by layers of Ba atoms. The ferroelectric transitions are above the melting temperatures of the compounds, while antiferromagnetic ordering takes place below $70 \mathrm{~K}$ (see Scott ${ }^{44}$ for a review).

Recently, the origin of ferroelectricity in $\mathrm{BaMF}_{4}$ has been studied using first principles calculations and it has been shown that ferroelectric displacements do not involve charge transfer between cations and anions; instead they arise due to size effects and geometrical constraints. Therefore, $\mathrm{BaMnF}_{4}$ has been classified as a geometric ferroelectric along with hexagonal manganites and ferrites. ${ }^{45}$

\subsection{3 $\quad M_{3} B_{7} O_{13} X$ boracites}

In these materials $\mathrm{M}$ is a divalent metal ion ranging from $\mathrm{Cr}$ to $\mathrm{Ni}$ in the $3 \mathrm{~d}$-series and $\mathrm{X}$ is a halogen $\mathrm{Cl}, \mathrm{Br}$, or $\mathrm{I}$. For most of these structures, the ferroelectric ordering takes place well above room temperature whereas the magnetic ordering (often weakly ferromagnetic) occurs at temperatures lower than $65 \mathrm{~K}$ (see Tolédano et al. ${ }^{46}$ for a review). Many of these materials undergo complex sequences of structural transitions on cooling. They possess large unit cells with many interatomic interactions; the structural complexity of the boracites has made it extremely difficult to obtain a clear picture of the factors causing ferroelectricity and the nature of the magnetoelectric coupling. 


\subsubsection{Organic-inorganic hybrids}

In the search for new multiferroic materials and mechanisms, organic-inorganic hybrids constitute an alternative. In hybrids, an inorganic transition metal halide constituent provides the magnetism and largely determines the electronic properties whereas a constituent comprised of organic molecules brings the advantage of easy processing and structural flexibility. These hybrids are a subgroup of metal-organic framework materials (MOFs). MOFs are an important class of materials and are extensively investigated for their use in applications such as gas storage, catalysis, drug delivery etc. Some organic-inorganic hybrids have been reported to exhibit multiferroic properties. ${ }^{47,48}$ These materials have a perovskite-like structure of composition $\mathrm{ABX}_{3}$, where $\mathrm{B}$ is a transition metal cation, $\mathrm{X}$ is a halide or small organic anion, and the voids are filled by a cationic organic molecule at the A site. ${ }^{49}$ $\left(\left(\mathrm{CH}_{3}\right)_{2} \mathrm{NH}_{2}\right) \mathrm{Mn}(\mathrm{HCOO})_{3}$ where $\mathrm{A}=\left[\left(\mathrm{CH}_{3}\right)_{2} \mathrm{NH}_{2}\right]^{+}, \mathrm{B}=\mathrm{Mn}^{2+}$ and $\mathrm{C}=\mathrm{HCOO}^{-}$is an example. This material orders antiferromagnetically below $8.4 \mathrm{~K}$ and has another structural transition manifested by a step-like decrease of the dielectric constant around $185 \mathrm{~K}$ on cooling. Thermal hysteresis is observed when the measurement is repeated on heating, suggesting a first-order phase transition. Temperature dependent X-ray diffraction analysis showed that an order-disorder-type phase transition from a high temperature rhombohedral paraelectric phase to a low temperature monoclinic ferroelectric phase coincided with the dielectric anomaly, rendering the material as a potential multiferroic. ${ }^{50}$ Recent theoretical calculations predict a ferroelectric polarization of $\sim 2 \mu \mathrm{C} \mathrm{cm}^{-2}$ for this compound, which can be increased up to $\sim 6 \mu \mathrm{C} \mathrm{cm}^{-2}$ by choosing different organic A cations. ${ }^{49}$

\subsubsection{Octahedral rotations and hybrid improper ferroelectricity}

The perovskite structure is a common crystal structure type that hosts many functional transition metal oxides including the proper ferroelectric $\mathrm{BaTiO}_{3}$ and colossal magnetoresistance oxides such as $\mathrm{La}_{1-\mathrm{x}} \mathrm{Ca}_{\mathrm{x}} \mathrm{MnO}_{3}$. It is represented as $\mathrm{ABO}_{3}$ where $\mathrm{A}$ is usually an alkaline earth or a rare earth ion and $\mathrm{B}$ is a transition metal ion octahedrally coordinated by oxygen ions. For ions having small ionic radius on the $\mathrm{A}$ site, $\mathrm{BO}_{6}$ octahedra collectively rotate in order to optimize the coordination of the A site cation. These rotations are known to be detrimental to ferroelectricity as most perovskites with octahedral rotations crystallize in non-polar space groups. Exceptions are materials with $\mathrm{Bi}^{3+}$ or $\mathrm{Pb}^{2+}$ on the A-site, having lone-pair ions inducing ferroelectric displacements. It has been recently shown that the inversion symmetry at the B-site can be broken by structural design via cation ordering on the A-site in layered perovskites such as Ruddlesden-Popper phases. Here, anti-polar displacements of the A-site cations in different layers of the perovskite do not cancel out, yielding a net polarization. ${ }^{51}$ This mechanism has been coined as hybrid improper ferroelectricity $^{52}$ as the ferroelectric polarization is induced by octahedral rotations of different symmetry in the different layers, thus the word 'hybrid.' The ferroelectricity is improper because the ferroelectric distortion is a by-product of the octahedral rotations, which is the primary order parameter. Experimental demonstration of hybrid improper ferroelectricity has been achieved in some Ruddlesden-Popper phases including $\mathrm{Ca}_{3} \mathrm{Ti}_{2} \mathrm{O}_{7}$ and Sr-doped $\mathrm{Ca}_{3} \mathrm{Ti}_{2} \mathrm{O}_{7}$, which are not magnetically ordered but exhibit relatively large spontaneous polarization $\left(8 \mu \mathrm{C} \mathrm{cm}^{-2}\right.$ for $\left.\mathrm{Ca}_{3} \mathrm{Ti}_{2} \mathrm{O}_{7}\right) .{ }^{53}$ Another example of hybrid improper ferroelectricity is the room temperature multiferroic $(1-\mathrm{x})\left(\mathrm{Ca}_{\mathrm{y}} \mathrm{Sr}_{1-\mathrm{y}}\right)_{1.15} \mathrm{~Tb}_{1.85} \mathrm{Fe}_{2} \mathrm{O}_{7-\mathrm{x}} \mathrm{Ca}_{3} \mathrm{Ti}_{2} \mathrm{O}_{7}$. Here, the structural design of octahedral rotations also tunes the magnetization to stabilize a weak ferromagnetic state in addition to the ferroelectric displacements induced by the same rotations. ${ }^{54}$ The recent mechanism of hybrid improper ferroelectricity shows potential for obtaining new multiferroic materials with enhanced functionalities.

\subsubsection{Charge order}

\subsubsection{Half-doped manganites}

Nearly half-doped rare earth manganites $\mathrm{R}_{1-\mathrm{x}} \mathrm{Ca}_{\mathrm{x}} \mathrm{MnO}_{3}$ ( $\mathrm{R}$ is the rare earth) show charge order: this can be interpreted as sitecentered charge order $(\mathrm{x}=0.5)$ where $\mathrm{Mn}^{4+}$ and $\mathrm{Mn}^{3+}$ sites are ordered in a checkerboard pattern or as bond-centered $(\mathrm{x}=0.4)$ charge ordering in which dimerization of two neighboring manganese sites renders bonds inequivalent, concentrating charge density between the nuclei. Inversion symmetry is retained for these particular charge ordering configurations. However, a charge ordering configuration that is intermediate between site-centered and bond-centered charge order is theoretically predicted to develop a net electronic polarization. ${ }^{55,56}$

Experimental studies have been complicated by the relatively high electrical conductivity of the doped manganites, screening the polarization. However, a study by Lopes et al. seems to show evidence for the possible development of polarization. ${ }^{57}$

\subsubsection{2 $\mathrm{LuFe}_{2} \mathrm{O}_{4}$}

$\mathrm{LuFe}_{2} \mathrm{O}_{4}$ was initially proposed as a potential multiferroic where the ferroelectric order is driven by charge order. ${ }^{58}$ This material is built from alternating stacked layers of lutetium oxide and iron oxide bilayers. The mixed-valent Fe ions are interconnected on a triangular mesh with inherent charge frustration. The originally proposed charge ordered superstructure consists of bilayers comprising stacked $\mathrm{Fe}^{2+}$-rich and $\mathrm{Fe}^{3+}$-rich layers and supports a polarization. An overall equal amount of $\mathrm{Fe}^{2+}$ and $\mathrm{Fe}^{3+}$ ions is retained.

The triangular geometry of the iron oxide layers complicates charge order within a single layer and charge separation between the layers of the bilayer stack is favored instead. $\mathrm{LuFe}_{2} \mathrm{O}_{4}$ shows charge order below $320 \mathrm{~K}$ and a net electric polarization is thought to develop across grouped pairs of $\mathrm{Fe}_{2} \mathrm{O}_{4}$ layers. Ferrimagnetic order follows at $\mathrm{T}_{\mathrm{N}}=240 \mathrm{~K}$ with a complex interplay between electric polarization and spin structure. However, the presence of ferroelectricity has not yet been unequivocally established. 
A recent study found a different charge ordered structure with non-polar bilayers ${ }^{59}$ and a resonant X-ray study by Lafuerza $e t$ al. ${ }^{60}$ has indicated that in fact no net polarization is present in the material.

For a detailed review of more charge order induced materials we refer the reader to. ${ }^{61,62}$

\subsection{Type II Multiferroics: Improper Ferroelectricity Induced by Spin Order}

The inversion symmetry of a material can be broken by magnetic order in various ways. ${ }^{63}$ We distinguish different microscopic magnetoelectric coupling mechanisms and discuss the details on the basis of a relevant material. Materials showing improper ferroelectricity, which arises as a consequence of spin order, can be subdivided into three groups based on distinct magnetoelectric coupling mechanisms. The first group consists of materials with collinear magnetic order, in which electrical polarization is induced by symmetric exchange striction. The second group of materials shows non-collinear magnetic order and supports electrical polarization due to anti-symmetric exchange striction. The polarization is driven by weaker relativistic spin-orbit coupling interactions, resulting in a smaller electrical polarization compared to that of the first group. The third class includes materials with electrical polarization induced by spin-dependent $\mathrm{p}$-d hybridization. In some materials, multiple coupling mechanisms are active. For an extensive review on multiferroics of spin origin, we refer the reader to Tokura et al. ${ }^{63}$

\subsubsection{Cycloidal spin spirals $\left(\mathrm{RMnO}_{3}\right)$}

The discovery of ferroelectricity resulting from competing magnetic interactions in the perovskites $\mathrm{TbMnO}_{3} \mathrm{and}_{\mathrm{DyMnO}}{ }^{16,64}$ has attracted much attention and has opened the field to a completely different approach to generating ferroelectricity in oxides. These materials were previously known as orthorhombic compounds with Pnma (Pbnm) space group symmetry. This space group exhibits inversion symmetry and thus no ferroelectric order was expected. In orthorhombic manganites, the $\mathrm{GdFeO}_{3}$ type distortion increases for smaller rare earth ionic radius, changing the Mn-O-Mn superexchange angle. An incommensurate cycloidal spin-spiral phase lying between regions of the phase diagram with collinear A-type (larger R such as La, Pr) and E-type AFM (smaller R such as Ho, Tm) order is stabilized. Polarization in this incommensurate AFM region was first observed in $\mathrm{TbMnO}_{3}$ by Kimura et al. in $2003^{16}$ and was later associated with the cycloidal spiral phase in a neutron diffraction study. ${ }^{65}$ Competing ferromagnetic nearest-neighbor and antiferromagnetic next-nearest-neighbor interactions stabilize the spin spiral state that drives the polarization. Figure 5(a) shows a cycloidal spin spiral with spins rotating in a plane defined by the normal vector $\vec{e}$. The spin spiral is incommensurate with the crystal structure and propagates along direction $\vec{Q}$. The pitch of the spin spiral depends on the respective magnitudes of the competing exchange interactions. As a consequence of the incommensurate spin spiral magnetic order, an electric dipole moment develops in the spin rotation plane (here the ab plane), perpendicular to the direction of the spin chain. ${ }^{66}$ Microscopically, the Dzyaloshinskii-Moriya (DM) interaction $\vec{D} \cdot \overrightarrow{S_{1}} \times \overrightarrow{S_{2}}{ }^{67,68}$ has a propensity to drive spins at right angles favouring non-collinear spin ordering and is governed by relativistic spin-orbit coupling interactions. Conversely, the inverse Dzyaloshinskii-Moriya (DM) interaction ${ }^{69}$ involves the magnetostrictive displacement $\vec{x}$ of oxygen ligands perpendicular to the spin chain shown in Figure $5(\mathrm{~b})$ as a black line in order to relax the lattice energy while adapting to the spin spiral configuration. The relatively small polarization in $\mathrm{TbMnO}_{3}$ is induced by very small polar lattice displacements that are beyond the resolution of conventional diffraction techniques, but Walker et al. have employed special techniques ${ }^{70}$ to resolve the polar lattice shifts in $\mathrm{TbMnO}_{3}$ with femtoscale accuracy. As shown in Figure 5(b), for cycloidal spirals the Dzyaloshinskii vector $\vec{D}$ is proportional to the cross product of the vector $\overrightarrow{r_{i j}}$ connecting neighboring spins and the displacement of the intervening oxygen ligand $\vec{x}$. The energy gain due to the DM interaction is $\vec{D} \cdot \overrightarrow{S_{1}} \times \overrightarrow{S_{2}}$ and therefore effectively displaces the oxygen ligands sideways perpendicular to the chain (along $\vec{x}$ ) and a polarization $\vec{P} \propto \overrightarrow{e_{i j}} \times \overrightarrow{S_{i}} \times \overrightarrow{S_{j}}$ develops. This concept was experimentally demonstrated for $\mathrm{TbMnO}_{3}$ where the spin helicity changes in an applied electric field. ${ }^{71}$ Alternatively, the net polarization can be explained as a purely electronic effect, without invoking ion displacements, in terms of a spin current ${ }^{72}$ where one spin precesses in the local field of the neighboring spin. Notably, the solid solutions $\mathrm{Eu}_{1-\mathrm{x}} \mathrm{Y}_{\mathrm{x}} \mathrm{MnO}_{3}$ and $\mathrm{Gd}_{1-\mathrm{x}} \mathrm{Tb}_{\mathrm{x}} \mathrm{MnO}_{3}{ }^{73}$ show spin spirals in the ab plane, whereas in $\mathrm{TbMnO}_{3}$ and $\mathrm{DyMnO}_{3}$ the spirals are confined to the bc plane. ${ }^{65,74}$ The complex interplay between single-ion anisotropy and the Dzyaloshinskii-Moriya interaction determines which spin cycloid rotation plane is favored. Specifically, the single-ion anisotropy defines the c-axis as the hard magnetization axis and would stabilize an ab-plane cycloid spiral with polarization along the $\mathrm{c}$ axis. The Dzyaloshinskii vector of the out-of-plane bonds stabilizes the ab-plane cycloid. ${ }^{75}$ The spin rotation plane can be changed in a polarization flop transition, ${ }^{16}$ where the orientation of the polarization is controlled by an applied magnetic field. Cycloidal spirals have been modeled extensively in theoretical studies and most of the phases found in orthorhombic manganites are now understood. ${ }^{73}$

Spin spirals typically appear at low temperatures due to magnetic frustration. Cycloidal spin spiral phases are often preceded by a para-electric phase with commensurate sinusoidal spin ordering. Nevertheless, $\mathrm{CuO}$ shows spin spiral order between 230 and $213 \mathrm{~K}^{76}$ and Kitagawa et al. reported a magnetoelectric spiral at room temperature in the hexaferrite $\mathrm{Sr}_{3} \mathrm{Co}_{2} \mathrm{Fe}_{24} \mathrm{O}_{41}{ }^{77} \mathrm{Additionally}$, only moderate magnetic fields (approximately $0.2 \mathrm{~T}$ ) are required to control the polarization making it a potential candidate for device applications. 
a)

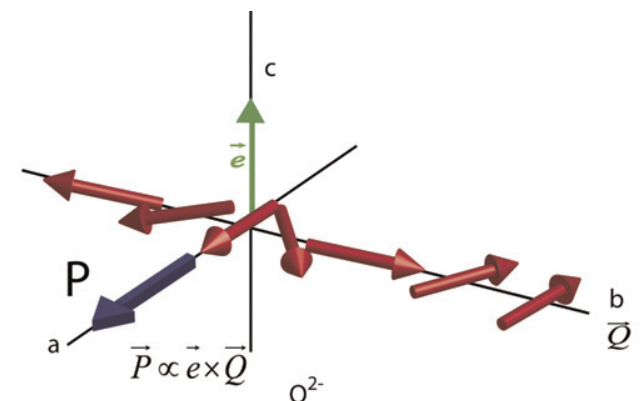

b)

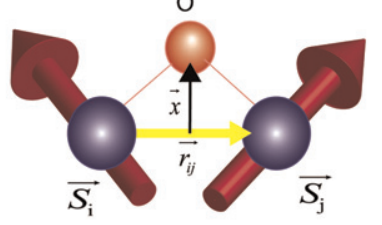

c)

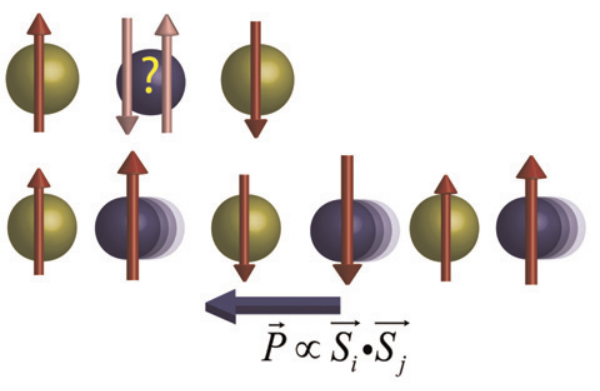

e) d)

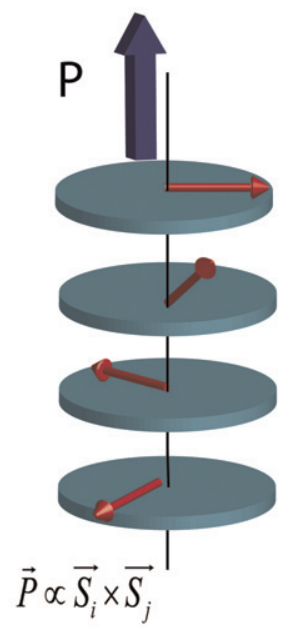

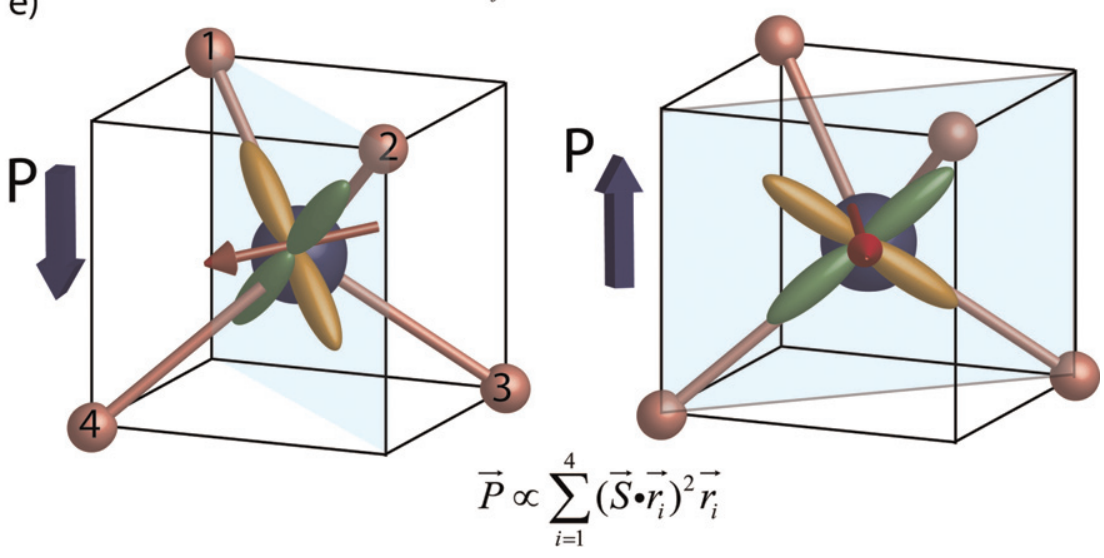

Figure 5 (a) Spins, shown in red, rotate along vector $e$ (green) with a spiral order propagating along $Q$. This results in a net polarization that develops perpendicular to the spin chain. (b) The Dzyaloshinskii-Moriya interaction between neighboring spins $\mathrm{S}_{\mathrm{i}}$ and $\mathrm{S}_{\mathrm{j}}$. The displacement $\vec{x}$ of the intervening oxygen ligand and the separation between the neighboring spins $S_{1}$ and $S_{2}$ determine the magnitude of the Dzyaloshinskii vector $\vec{D}$. (c) Symmetric exchange striction in collinear magnets. Purple spheres are $\mathrm{Mn}^{3+}(\mathrm{S}=2)$ and yellow spheres are $\mathrm{Mn}^{4+}(\mathrm{S}=3 / 2)$. Parallel spin alignment results in shorter distances between spins. Antiparallel alignment results in longer bonds. (d) Proper screw spin order with net polarization shown in purple parallel to propagation direction of the spin helix. Green circles are envelopes of spin rotation. (e) $\pi$-Bonding between oxygen ligand and transition metal $3 \mathrm{~d}$ orbital is affected by the spin orientation of the transition metal. The spin driven polarization creates a local dipole moment.

\subsection{2 p-d Hybridization}

$\mathrm{Ba}_{2} \mathrm{CoGe}_{2} \mathrm{O}_{7}$ adopts the tetragonal, non-centrosymmetric apolar åkermanite structure in which cobalt ions are tetrahedrally coordinated by the surrounding oxygen ligands as shown in Figure 5(e). Spin dependent p-d hybridization of the $\mathrm{Co}^{2+} 3 \mathrm{~d}$ orbitals with the p-orbitals of the oxygen ligands renders the hybridization asymmetric, creating different local dipole moments along the Co-O bonds. This magnetoelectric coupling mechanism relies on a single localized spin coordinated to a ligand and is mediated via spin-orbit coupling. ${ }^{78,79}$ The polarization is proportional to $\vec{P} \propto\left(\vec{S} \cdot \overrightarrow{r_{i}}\right)^{2} \overrightarrow{r_{i}}$, where $\vec{S}$ is the local spin and $\overrightarrow{r_{i}}$ denotes the vector describing the metal-ligand bond.

$\mathrm{Ba}_{2} \mathrm{CoGe}_{2} \mathrm{O}_{7}$ orders in a commensurate magnetic (ground state) structure below $\mathrm{T}_{\mathrm{N}}=6.7 \mathrm{~K}^{80}$ in which the antiferromagnetic spins are confined to the tetragonal $(\mathrm{ab})$ plane and with a weak in-plane ferromagnetic component ${ }^{81}$ and a concomitant 
polarization. ${ }^{82}$ The microscopic coupling mechanism was discussed later by Murakawa et al. ${ }^{83}$ On rotating the spins in the basal plane, a charge density redistribution takes place between the upper pair of oxygens (oxygens 1,2 in Figure 5(e)) and the lower pair of oxygens (oxygens 3,4 in Figure 5(e)) corresponding to an orbital admixture of the different $\mathrm{d}_{\mathrm{yz}} \mathrm{d}_{\mathrm{zx}}$ orbitals ${ }^{84}$ due to spin-orbit coupling (the occupied states are mainly of $\mathrm{d}_{\mathrm{x}-\mathrm{y}}^{2}{ }^{2}$ and $\mathrm{d}_{3 \mathrm{z}-\mathrm{r}}^{2}{ }^{2}$ character). The spin direction is perpendicular to the principal plane of the relevant orbital (light blue in Figure 5(e)). When the spin orientation is parallel to the upper O1-O2 pair, the lower bonds stretch, resulting in an upward polarization. Spin-orbit coupling lifts the degeneracies of the $\mathrm{t}_{2 \mathrm{~g}}$ and $\mathrm{e}_{\mathrm{g}}$ orbital sets. ${ }^{84}$ The polarization along $\mathrm{c}$ follows a sinusoidal modulation with the angle at which the magnetic field is rotated around the $\mathrm{z}$ axis. When a magnetic field is applied along the ${ }^{110}$ direction Pc is maximum. The polarization crosses through zero when the magnetic field is parallel to the a or b axis. ${ }^{83}$

A detailed analysis based on symmetry considerations has described how $\mathrm{Ba}_{2} \mathrm{CoGe}_{2} \mathrm{O}_{7}$ cycles through a series of magnetic point groups when the magnetic field is rotated and provides a phenomenological account for the experimentally observed behaviour. ${ }^{83,85}$ The magnetoelectric effect in this material has also been attributed to the presence of a toroidal moment. ${ }^{86}$

\subsubsection{Proper screw spin order}

Materials with proper screw spin order $(\vec{Q} / / \vec{e})$, as shown in Figure 5(d), develop a polarization parallel to the spin propagation direction of the spiral, in stark contrast to the cycloidal spiral multiferroics discussed earlier. The observed polarization in these materials cannot be explained by the standard spin current model as discussed for the cycloidal spiral phase. The parent phase with this type of spin order, the delafossite $\mathrm{CuFeO}_{2}$, shows a multitude of magnetic phases in applied fields ${ }^{87}$ below its ordering temperature of $14 \mathrm{~K}$. In the ground state below $10.5 \mathrm{~K}$, a commensurate up-up-down-down magnetic phase is stabilized ${ }^{88}$ that is retained in fields up to $7 \mathrm{~T}$. When a magnetic field is applied in the c direction, multiple field induced transitions are observed. ${ }^{87}$ The first field-induced phase is stable in magnetic fields between 7 and $13.5 \mathrm{~T},{ }^{89}$ and is an incommensurate ferroelectric phase ${ }^{87,89}$ that shows proper screw order. ${ }^{90}$ Partial chemical substitution of $\mathrm{Fe}$ by $\mathrm{Al}^{3+}$ or $^{\mathrm{Ga}}{ }^{3+91}$ stabilizes the proper screw helical phase in zero magnetic field. ${ }^{92}$

The observed polarization in these proper screw magnets can be explained in terms of a spin-dependent metal-ligand p-d hybridization $^{78,79,93}$ mechanism. Alternatively, Kaplan et al. proposed an extension of the inverse Dzyaloshinskii-Moriya mechanism ${ }^{94}$ with a polarization proportional to the spin twist $S_{i} \times S_{j}$. Here, there is an additional orthogonal term in the Dzyaloshinskii vector that was previously not taken into account, as pointed out by Kaplan and Mahanti. ${ }^{94}$ The inverse Dzyaloshinskii-Moriya effect is also active here, analogous to the cycloidal spin spiral.

The polarization that develops parallel to the propagation vector of the spin helix has been measured as $300 \mu \mathrm{C} \mathrm{m}{ }^{-2} .89 \mathrm{~A}$ soft X-ray resonant scattering study of superlattice reflections at the $\mathrm{Fe} \mathrm{L}_{2,3}$ absorption edge has shown that orbital order of the $\mathrm{t}_{2 \mathrm{~g}}$ state is stabilised by spin-orbit coupling. The orbital order causes a modulation of the metal-ligand hybridization, corroborating the proposed $\mathrm{p}-\mathrm{d}$ spin dependent hybridization coupling mechanism. ${ }^{95}$

\subsubsection{Ferroaxial multiferroics $\left(\mathrm{CaMn}_{7} \mathrm{O}_{12}\right)$}

In the trigonal quadruple perovskite $\mathrm{CaMn}_{7} \mathrm{O}_{12}{ }^{96-98}$ the octahedrally coordinated $\mathrm{Mn}^{3+}$ sites are susceptible to a structural JahnTeller distortion that deforms the oxygen octahedra. Incommensurate orbital order sets in at $250 \mathrm{~K}$ with a concomitant incommensurate superstructure modulation associated with a ferro-axial distortion in which the orbitals rotate continuously along the threefold axis. Ferro-axial materials support a distinguishable clockwise or counterclockwise sense of rotation of a structural element about an axial vector $\vec{A}$ with respect to the rest of the crystal structure. The nature of the Heisenberg symmetric superexchange interactions is dictated by the Goodenough-Kanamori-Anderson rules ${ }^{99}$ and the orbital modulation stabilizes a proper screw helical magnetic structure. The specific orbital order in $\mathrm{CaMn}_{7} \mathrm{O}_{12}$ affects the superexchange interactions when the material orders below $\mathrm{T}_{\mathrm{N} 1}=90 \mathrm{~K}$ and renders the magnetic structure chiral. The chiral magnetic order with helicity $\sigma$ couples with the structural axiality to generate a polarization $\vec{P}=\gamma \sigma \vec{A}$. This ferro-axial coupling mechanism is different from the magnetoelectric coupling mechanism in achiral ${ }^{100}$ cycloidal spin spirals, although again polar lattice shifts are induced via the inverse Dzyaloshinskii-Moriya mechanism. The polarization of $2870 \mu \mathrm{C} \mathrm{m}^{-2}$ observed in $\mathrm{CaMn}_{7} \mathrm{O}_{12}$ is unprecedented for improper ferroelectric multiferroics driven by magnetic helicity and is the combined effect of the inverse Dzyaloshinskii-Moriya mechanism and symmetric exchange striction. ${ }^{101}$

\subsubsection{Symmetric exchange striction}

5.2.5.1 $\mathrm{Ca}_{3} \mathrm{CO}_{2-x} \mathrm{Mn}_{x} \mathrm{O}_{6}$

In symmetric exchange striction, the neighboring spins shift in order to optimize their exchange energy. The material $\mathrm{Ca}_{3} \mathrm{Co}_{2-\mathrm{x}} \mathrm{Mn}_{\mathrm{x}} \mathrm{O}_{6}(\mathrm{x} \sim 0.96)$ epitomizes this type of magnetostriction. This mechanism was proposed earlier by Chapon ${ }^{102}$ and relies on symmetric superexchange instead of spin-orbit coupling.

The $\mathrm{Ca}_{3} \mathrm{Co}_{2} \mathrm{O}_{6}$ crystal structure contains pillars of alternating face-sharing $\mathrm{CoO}_{6}$ trigonal prisms and octahedra. Mn has a strong preference for octahedral coordination and the parent $\mathrm{Ca}_{3} \mathrm{Co}_{2} \mathrm{O}_{6}$ phase can accommodate Mn on half of its octahedral sites. This results in an alternating chain of $\mathrm{Co}^{2+}$ and $\mathrm{Mn}^{4+}$. This magnetically active Ising chain orders below $16.5 \mathrm{~K}$. Competing ferromagnetic and antiferromagnetic nearest neighbor superexchange interactions stabilize an up-up-down-down ( $\uparrow \downarrow \downarrow)$ pattern as an 


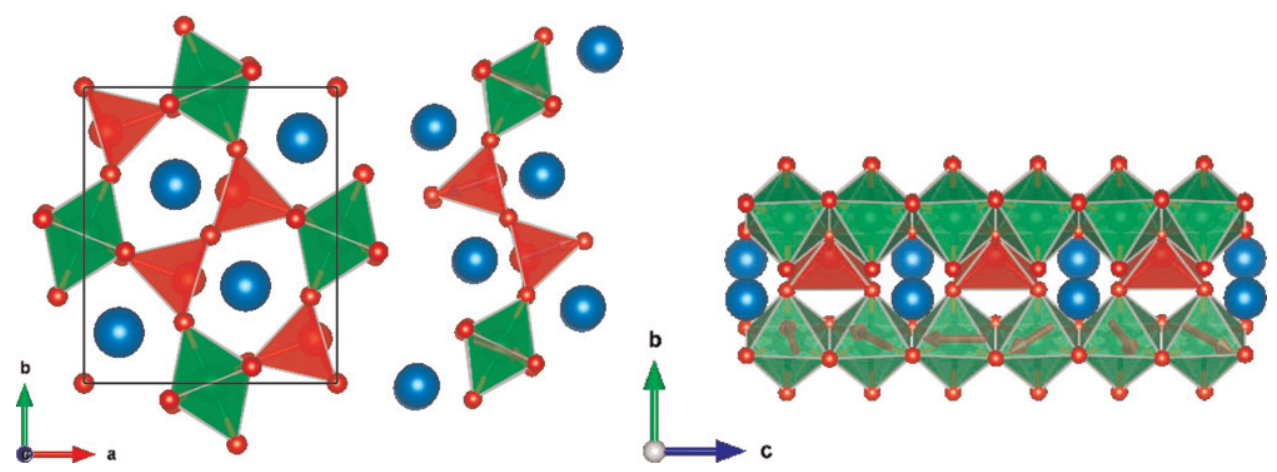

Figure 6 Structure of $\mathrm{RMn}_{2} \mathrm{O}_{5}: \mathrm{Mn}^{3+} \mathrm{O}_{5}$ pyramids shown in red, edge sharing $\mathrm{Mn}^{4+} \mathrm{O}_{6}$ octahedra shown in green. (Left) Zig-zag chain of Mn spins in ab plane. (Middle) magnetic $\uparrow \uparrow \downarrow \uparrow \mathrm{Mn}^{4+}-\mathrm{Mn}^{3+}-\mathrm{Mn}^{3+}-\mathrm{Mn}^{4+}$ chain running parallel to $\mathrm{b}$ axis. (Right) Cycloidal spiral in edge-sharing octahedra of the LT-ICM phase propagating along $\mathrm{C}$.

Ising chain magnet that consists of linear chains of $\mathrm{Co}^{2+}$ and $\mathrm{Mn}^{4+}$ ions. ${ }^{103,104}$ The $\mathrm{Co}^{2+}$ trigonal crystal field and spin-orbit coupling results in a large $\mathrm{Co}^{2+}$ magnetic moment of $1.7 \mu_{\mathrm{B}}$ and strong Ising behavior that keeps the magnetic moment rigidly along the spin chain direction. ${ }^{105}$

Figure 5(c) shows that parallel neigbouring spins that tend to shorten the bond length, whereas an antiparallel spin alignement leads to bond extension. A polarization proportional to $S_{i} \bullet S_{j}$ develops concomitantly with the magnetic ordering along the chain with a magnitude of $90 \mu \mathrm{C} \mathrm{m}^{-2}$ at $2 \mathrm{~K}$, which is suppressed if a magnetic field is applied along the c axis.

\subsubsection{2 $\mathrm{RMn}_{2} \mathrm{O}_{5}$}

$\mathrm{RMn}_{2} \mathrm{O}_{5}$ contains a zig-zag chain of inequivalent $\mathrm{Mn}^{3+}(\mathrm{S}=2)$ and $\mathrm{Mn}^{4+}(\mathrm{S}=3 / 2)$ sites along the b axis with $\mathrm{Mn}^{4+} \mathrm{O}_{6}$ octahedra and $\mathrm{Mn}^{3+} \mathrm{O}_{5}$ square pyramids, shown in Figure 6. $\mathrm{YMn}_{2} \mathrm{O}_{5}$ shows three magnetic structures when cooled down. The first magnetic phase is a para-electric incommensurate (ICM-HT) phase that orders below $45 \mathrm{~K}$. The first ferroelectric phase appears at $38 \mathrm{~K}$ with a concomitant commensurate (CM) antiferromagnetic order. A second incommensurate phase (ICM-LT) appears below $20 \mathrm{~K}$ with an additional cycloidal spin spiral ${ }^{106}$ order that propagates along the linear chain of edge sharing $\mathrm{Mn}^{4+}$ octahedra that runs parallel to c, as shown in the right panel of Figure $6 .{ }^{107}$

The improper ferroelectricity in the collinear $(\mathrm{CM})$ phase can be explained in terms of symmetric exchange striction effects. ${ }^{102} \mathrm{~A}$ magnetically frustrated spin chain runs along the $\mathrm{b}$ axis and consists of pairs of almost parallel and antiparallel $\mathrm{Mn}^{3+}$ and $\mathrm{Mn}^{4+}$ spins with $\uparrow \uparrow \downarrow \uparrow$ order, as shown schematically in the central panel of Figure 6 . In this chain, exchange energy is gained by a polar lattice distortion in which the bond distance between antiparallel spins shrinks and the bond distance between parallel oriented spins expands. The collective magnetostrictive shifts result in a polarization of $\sim 1100 \mu \mathrm{C} \mathrm{m}^{-2} 108$ that develops along the $\mathrm{b}$ axis.

The improper ferroelectricity of $\mathrm{YMn}_{2} \mathrm{O}_{5}$ has dual nature, originating from both inverse Dzyaloshinskii-Moriya interactions associated with the cycloidal spin spiral along the c-axis and from symmetric exchange striction associated with collinear spin order. Wakimoto et al. ${ }^{107}$ selectively replaced $\mathrm{Mn}^{3+}$ in the host lattice by $\mathrm{Ga}^{3+}$, disrupting the AFM zig-zag chain and destroying the $\mathrm{CM}$ order for $12 \%$ substitution. However, they did observe a polarization below $20 \mathrm{~K}$, where the cycloidal spin phase appears. For an overview of other rare earth $\mathrm{RMn}_{2} \mathrm{O}_{5}$ compounds we refer the reader to Kimura et al. ${ }^{109}$

\subsubsection{E-type $\mathrm{RMnO}_{3}$}

Rare-earth manganites with small rare earth radii are typically most stable in the hexagonal structure. However, the orthorhombic perovskite phase can be stabilised with high pressure hydrothermal synthesis methods ${ }^{110}$ and these materials show collinear E-type ordering in the ab plane with zig-zag chains, as shown in Figure 7. Exchange striction causes the intervening oxygen ligands to shift and induces local dipole moments as shown by the blue arrows in Figure 7. E-type collinear orthorhombic manganites are predicted to show considerably larger polarization compared to those adopting the cycloidal spin-spiral order. ${ }^{11,112}$ Polarization has been confirmed experimentally for $\mathrm{HoMnO}_{3},{ }^{113,114}$ DyMnO$_{3}{ }^{115}$ and $\mathrm{Tb}_{1-\mathrm{x}} \mathrm{Ho}_{\mathrm{x}} \mathrm{MnO}_{3}{ }^{116}$ which shows optimum polarization for $0.2<\mathrm{x}<0.4$. The polarization reaches $1500 \mu \mathrm{C} \mathrm{m}^{-2}$ in orthorhombic $\mathrm{TmMnO}_{3}{ }^{117}$ and even $2200 \mu \mathrm{C} \mathrm{m}^{-2}$ for $\mathrm{YMnO}_{3}{ }^{110} \mathrm{For} \mathrm{a}$ general overview of all E-type multiferroics (and cycloidal spirals) see the review of Ishiwata et al. ${ }^{118}$

\section{Single Phase Multiferroic Thin Films}

Hexagonal manganite films were among the first multiferroic single phase thin film systems to be studied. These systems have served as excellent model systems for growth studies and strain engineering has allowed the stabilization of phases that would otherwise require high pressure synthesis in bulk. However, the low ordering temperatures and small polarization values have 


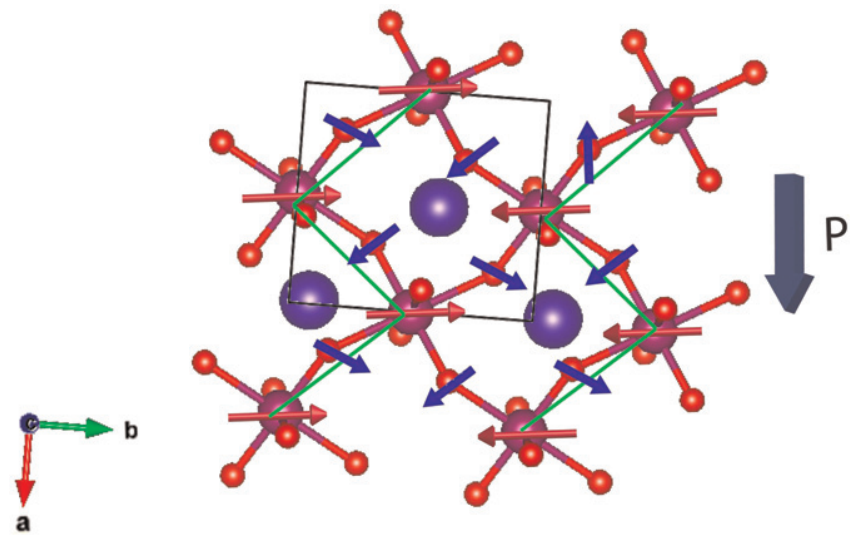

Figure 7 ab plane of $\mathrm{RMnO}_{3}$ with E-type order in zig-zag chains shown by green line. The local dipoles due to exchange striction are shown in blue. Mn atoms are shown in purple, oxygen ions in red and rare earth atoms in blue.

a)

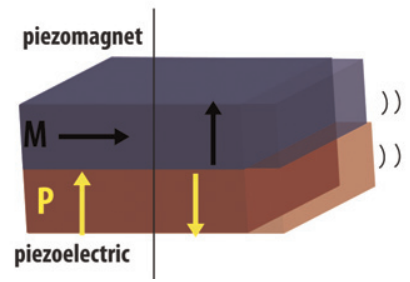

b)

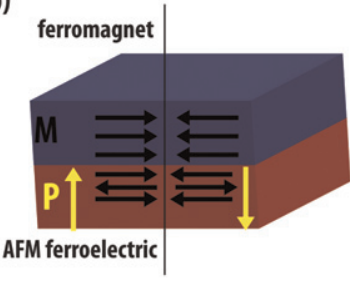

c)

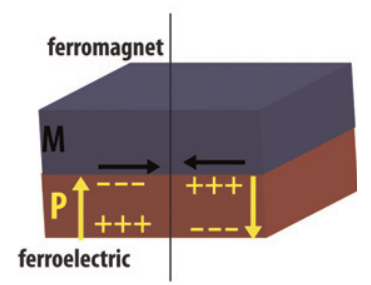

Figure 8 Magnetoelectric coupling mechanisms in artificial multiferroics. Vertical lines delineate moments before and after a switching event. (a) Magnetoelectric coupling via elastic coupling between a piezomagnet and piezoelectric. (b) Exchange bias effect, in which a magnetoelectric antiferromagnet biases the magnetization of the ferromagnet via uncompensated spins at the interface. (c) Interface-charge-mediated magnetoelectric coupling. Spin density accumulates in the ferromagnet within the Thomas-Fermi screening length at the interface to shield bound interface charges induced by a ferroelectric in the charge-mediated magnetoelectric effect, inducing surface magnetization.

limited practical applications. For an extensive review on single-phase $\mathrm{RMnO}_{3}$ hexagonal and orthorhombic thin films we refer the reader to. ${ }^{119}$

$\mathrm{BiFeO}_{3}$ (BFO) is one of the most studied thin film multiferroic single phase materials. ${ }^{22,120}$ The bulk phase orders ferroelectrically at $1143 \mathrm{~K}$ and magnetically at $670 \mathrm{~K}$ as a G-type antiferromagnet with a superimposed incommensurate spin spiral that cancels out weak ferromagnetism due to spin canting, precluding linear magnetoelectric coupling. ${ }^{121}$ The polarization was measured to be an order of magnitude higher than that observed in bulk until much later drastic improvements were made to the quality of bulk samples ${ }^{24}$ and the measured polarization approached the same value. With strain engineering a unique tetragonallike phase can be stabilised under highly compressive strain that does not exist in bulk. Under these strain conditions BFO shows an enhanced polarization and a higher piezoelectric response than lead zirconate titanate (PZT), ${ }^{120}$ possibly providing a lead-free alternative to the conventionally used piezoelectric material PZT. Martin et al. have published a detailed review on BFO and $\mathrm{BiMnO}_{3}{ }^{122}$ For more information on multiferroic thin films, we refer the reader to the review papers. ${ }^{121-123}$

\section{Composite Structures}

Composite materials such as laminates ${ }^{124}$ or epitaxial heterostructures combine piezomagnetic (magnetostrictive) and piezoelectric materials that are elastically coupled via strain that is transmitted across the interface. The magnetoelectric coupling is the result of the combination of the magnetostrictive and piezoelectric properties of the individual phases, which are elastically coupled and are typically much larger than those found in single phase multiferroics. ${ }^{125}$ As shown in Figure 8, strain develops in response to an applied electric field which is transmitted across the interface, affecting the magnetization or magnetic anisotropy of the piezomagnetic material.

Nanoscale pillar structures of the magnetic spinel $\mathrm{CoFe}_{2} \mathrm{O}_{4}$ embedded in a matrix of the ferroelectric perovskite $\mathrm{BiFeO}_{3}$ combine a large effective interface area and avoid mechanical clamping to the substrate ${ }^{126,127}$ in order to further improve the coupling strength. This structure requires a small magnetic bias to lift the degeneracy between up and down magnetic states and to 
ensure electric field controlled magnetization switching. However, strain-mediated magnetoelectric coupling cannot rotate the magnetization over a full $180^{\circ},{ }^{128}$ which is a requirement for deterministic switching in memory device structures. ${ }^{129}$

\section{Interfacial Magnetoelectric Coupling with Electronic Effects in Multiferroic Heterostructures}

As an alternative to the elastic coupling of individual layers in heterostructures via strain, electronic surface effects can also be harnessed to mediate interfacial magnetoelectric coupling. ${ }^{128,130}$ The exchange bias effect, as shown in Figure 8(b), mediates interfacial coupling via inter-layer exchange interactions in a bilayer system of a ferromagnet and antiferromagnet, in which the uncompensated spins of the antiferromagnet are coupled to the ferromagnetic layer. Polarization biases the orientation of the ferromagnet and causes the $\mathrm{M}-\mathrm{H}$ hysteresis loop to shift horizontally. Further research is necessary to fully understand the microscopic mechanisms involved. This electric field controlled magnetization switching mechanism has been demonstrated in $\mathrm{Pd} / \mathrm{Co} / \mathrm{Cr}_{2} \mathrm{O}_{3}{ }^{131}$ where the linear magnetolectric effect in $\mathrm{Cr}_{2} \mathrm{O}_{3}$ induces a small magnetization in response to an applied electric field.

Magnetoelectric coupling can also be mediated via an interface-charge-mediated mechanism, ${ }^{132}$ shown in Figure 8(c). The polarization of the ferroelectric material causes a build-up of bound interface charge. The ferromagnet screens this interface charge and this causes a local modulation of carrier density in the ferromagnet. Screening occurs over a very short range, confined to the interface within the Thomas-Fermi screening length of the metal. The exchange interaction in the ferromagnet splits the conduction band into spin-up and spin-down bands and consequently, the density of states for the spin-up and the spin-down bands at the Fermi level become different, affecting the spin imbalance at the Fermi level. Therefore, the screening interactions at the interface become spin-dependent, affecting locally the surface magnetization and the magnetic anisotropy. ${ }^{133}$ For a detailed review of more interfacial electronic coupling effects we refer the reader to ${ }^{125,128,130}$ and references therein.

\section{Multiferroic Domain Walls and Emergent Functional Properties at the Domain Walls}

Ferroic materials, i.e., ferroelectrics, ferromagnetic and ferroelastics, contain regions of differently orientated order parameters - for example, ferroelectric polarization in ferroelectrics - called domains. Domains are separated by domain walls, which are only a a few nanometers in size in ferroelectrics while magnetic domain walls are at least one order of magnitude wider. Domain walls can possess different functional properties than the domains themselves and control of these functionalities might be exploited in device applications. For example, the ferroelectric domain walls of multiferroic $\mathrm{BiFeO}_{3}$ are electrically conducting whereas the domains are insulating/semiconducting. ${ }^{134}$ The domain walls of an antiferromagnetic hexagonal manganite, ErMnO 3 were shown to possess a net spontaneous magnetization. ${ }^{135}$ The multiferroic domain walls of hexagonal manganites are very complex. As explained in Section 5.1.2, in these improper ferroelectric materials ferroelectricity appears as a by-product of a structural phase transition. Structural antiphase boundaries appear below this transition and they also set up the ferroelectric domains. At still lower temperatures around $100 \mathrm{~K}$, antiferromagnetic domains form, pinned by the ferroelectric domains. ${ }^{35}$ The coupling of structural, ferroelectric and magnetic domains gives rise to the emergence of topologically protected domain walls. ${ }^{136}$ In-depth information on the properties and potential applications of multiferroic domain walls can be found in refs. ${ }^{137-139}$.

\section{References}

1. Smolenskii, G.A., Chupis, I.E., 1982. Segnetomagnetics. Uspekhi Fizicheskikh Nauk 137 (3), 415-448.

2. Khomskii, D., 2009. Classifying multiferroics: Mechanisms and effects. Physics 2, 20.

3. Cheong, S.W., Mostovoy, M., 2007. Multiferroics: A magnetic twist for ferroelectricity. Nature Materials 6 (1), 13-20.

4. Fiebig, M., 2005. Revival of the magnetoelectric effect. Journal of Physics D-Applied Physics 38 (8), R123-R152.

5. Eerenstein, W., Mathur, N.D., Scott, J.F., 2006. Multiferroic and magnetoelectric materials. Nature 442 (7104), 759-765.

6. Scott, J.F., 2007. Data storage - Multiferroic memories. Nature Materials 6 (4), 256-257.

7. Scott, J.F., 2012. Applications of magnetoelectrics. Journal of Materials Chemistry 22 (11), 4567-4574.

8. Bibes, M., Barthelemy, A., 2008. Multiferroics: Towards a magnetoelectric memory. Nature Materials 7 (6), 425-426.

9. Quindeau, A., et al., 2015. Four-state ferroelectric spin-valve. Scientific Reports 5, 09749.

10. Pantel, D., Goetze, S., Hesse, D., Alexe, M., 2012. Reversible electrical switching of spin polarization in multiferroic tunnel junctions. Nature Materials 11 (4), 289-293.

11. Gajek, M., et al., 2007. Tunnel junctions with multiferroic barriers. Nature Materials 6 (4), 296-302.

12. Tsymbal, E.Y., Kohlstedt, H., 2006. Applied physics - tunneling across a ferroelectric. Science 313 (5784), 181-183.

13. Julliere, M., 1975. Tunneling between ferromagnetic films. Physics Letters A 54 (3), 225-226.

14. Tsymbal, E.Y., Gruverman, A., Garcia, V., Bibes, M., Barthelemy, A., 2012. Ferroelectric and multiferroic tunnel junctions. MRS Bulletin 37 (2), $138-143$.

15. Huai, Y.M., Albert, F., Nguyen, P., Pakala, M., Valet, T., 2004. Observation of spin-transfer switching in deep submicron-sized and low-resistance magnetic tunnel junctions. Applied Physics Letters 84 (16), 3118-3120.

16. Kimura, T., et al., 2003. Magnetic control of ferroelectric polarization. Nature 426 (6962), 55-58.

17. Hill, N.A., 2000. Why are there so few magnetic ferroelectrics? Journal of Physical Chemistry B 104 (29), 6694-6709.

18. Kittel, C., 1996. Introduction to Solid State Physics, seventh ed. New York: Wiley.

19. Lines, M.E., Glass, A.M., 2001. Principles and Applications of Ferroelectrics and Related Materials. Oxford: Oxford University Press. 
20. Catalan, G., Scott, J.F., 2009. Physics and applications of bismuth ferrite. Advanced Materials 21 (24), 2463-2485.

21. Bokov, V.A., Myl'nikova, I.E., Smolenskii, G.A., 1962. Ferroelectric antiferromagnetics. Soviet Physics. JETP 15, 447.

22. Wang, J., et al., 2003. Epitaxial $\mathrm{BiFeO}_{3}$ multiferroic thin film heterostructures. Science 299 (5613), 1719-1722.

23. Rojac, T., Kosec, M., Budic, B., Setter, N., Damjanovic, D., 2010. Strong ferroelectric domain-wall pinning in $\mathrm{BiFeO}_{3}$ ceramics. Journal of Applied Physics 108 (7), 074107.

24. Lebeugle, D., et al., 2007. Room-temperature coexistence of large electric polarization and magnetic order in $\mathrm{BiFeO}_{3}$ single crystals. Physical Review B 76 (2), 024116.

25. Heron, J.T., et al., 2014. Deterministic switching of ferromagnetism at room temperature using an electric field. Nature 516 (7531), 370.

26. Katsufuji, T., Takagi, H., 2001. Coupling between magnetism and dielectric properties in quantum paraelectric EuTiO 3 . Physical Review B 64 (5), 054415.

27. Lee, J.H., et al., 2010. A strong ferroelectric ferromagnet created by means of spin-lattice coupling. Nature 466 (7309), 954.

28. van Aken, B.B., Meetsma, A., Palstra, T.T.M., 2001. Hexagonal $\mathrm{YMnO}_{3}$. Acta Crystallographica Section C-Crystal Structure Communications 57, $230-232$.

29. Abrahams, S.C., 2001. Ferroelectricity and structure in the $\mathrm{YMnO}_{3}$ family. Acta Crystallographica Section B-Structural Science $57,485-490$.

30. Bos, J.W.G., van Aken, B.B., Palstra, T.T.M., 2001. Site disorder induced hexagonal-orthorhombic transition in $\mathrm{Y}_{1-x} \mathrm{Gd}_{x} \mathrm{MnO}_{3}$. Chemistry of Materials 13 (12), 4804-4807.

31. Bertaut, F., Forrat, F., Fang, P., 1963. Les manganites de terres rares et d'yttrium: une nouvelle classe de ferroelectriques. C.R. Acad. Science 256, 1958.

32. Huang, Z.J., Cao, Y., Sun, Y.Y., Xue, Y.Y., Chu, C.W., 1997. Coupling between the ferroelectric and antiferromagnetic orders in YMnO 3 . Physical Review B 56 (5), 2623-2626.

33. Katsufuii, T., et al., 2001. Dielectric and magnetic anomalies and spin frustration in hexagonal $\mathrm{RMnO}_{3}(\mathrm{R}=\mathrm{Y}$, $\mathrm{Yb}$, and Lu). Physical Review $\mathrm{B} 64$ (10), 104419.

34. Fiebig, M., Lottermoser, T., Frohlich, D., Goltsev, A.V., Pisarev, R.V., 2002. Observation of coupled magnetic and electric domains. Nature 419 (6909), 818-820

35. Lottermoser, T., et al., 2004. Magnetic phase control by an electric field. Nature 430 (6999), 541-544.

36. Ismailzade, I.G., A., K.S., 1965. Determination of the curie point of the ferroelectrics $\mathrm{YMnO}_{3}$ and $\mathrm{YbMnO}_{3}$. Soviet Physics of the Solid State 7, 236-238.

37. Lukaszew., K., Karutkal., J., 1974. X-ray Investigations of crystal structure and phase transitions of YMnO . Ferroelectrics 7 (1-4), 81-82.

38. Lonkai, T., et al., 2004. Development of the high-temperature phase of hexagonal manganites. Physical Review B 69 (13), 134108.

39. Fennie, C.J., Rabe, K.M., 2005. Ferroelectric transition in $\mathrm{YMnO}_{3}$ from first principles. Physical Review B 72 (10), 100103.

40. Nénert, G., Ren, Y., Stokes, H.T., Palstra, T.T.M., 2005. Symmetry changes at the ferroelectric transition in the multiferroic YMnO3. cond-mat/0504546.

41. Gibbs, A.S., Knight, K.S., Lightfoot, P., 2011. High-temperature phase transitions of hexagonal YMnO3. Physical Review B 83 (9), 094111.

42. Xu, X.S., Wang, W.B., 2014. Multiferroic hexagonal ferrites (h-RFeO $3, R=Y$, Dy-Lu): A brief experimental review. Modern Physics Letters B 28 (21), 1430008.

43. Wang, W.B., et al., 2013. Room-Temperature Multiferroic Hexagonal $\mathrm{LuFeO}_{3}$ Films. Physical Review Letters 110 (23), 237601.

44. Scott, J.F., 1979. Phase transitions in BaMnF 4 . Reports on Progress in Physics 42 (6), 1055-1084.

45. Ederer, C., Spaldin, N.A., 2006. Origin of ferroelectricity in the multiferroic barium fluorides BaMF 4 : A first principles study. Physical Review B 74 (2), 024102.

46. Toledano, P., Schmid, H., Clin, M., Rivera, J.P., 1985. Theory of the low temperature phases in boracites - latent antiferromagnetism, weak ferromagnetism, and improper magnetostructural couplings. Physical Review B 32 (9), 6006-6038.

47. Stroppa, A., et al., 2011. Electric Control of magnetization and interplay between orbital ordering and ferroelectricity in a multiferroic metal-organic framework. Angewandte Chemie-International Edition 50 (26), 5847-5850

48. Polyakov, A.0., et al., 2012. Coexisting ferromagnetic and ferroelectric order in a $\mathrm{CuCl}_{4}$-based organic-inorganic hybrid. Chemistry of Materials 24 (1), 133-139.

49. Di Sante, D., Stroppa, A., Jain, P., Picozzi, S., 2013. Tuning the ferroelectric polarization in a multiferroic metal-organic framework. Journal of the American Chemical Society 135 (48), 18126-18130.

50. Jain, P., et al., 2009. Multiferroic behavior associated with an order-disorder hydrogen bonding transition in metal-organic frameworks (MOFs) with the Perovskite ABX Architecture. Journal of the American Chemical Society 131 (38), 13625-13627.

51. Benedek, N.A., Mulder, A.T., Fennie, C.J., 2012. Polar octahedral rotations: A path to new multifunctional materials. Journal of Solid State Chemistry 195, 11-20.

52. Benedek, N.A., Fennie, C.J., 2011. Hybrid improper ferroelectricity: A mechanism for controllable polarization-magnetization coupling. Physical Review Letters 106 (10), 107204.

53. Oh, Y.S., Luo, X., Huang, F.T., Wang, Y.Z., Cheong, S.W., 2015. Experimental demonstration of hybrid improper ferroelectricity and the presence of abundant charged walls in $(\mathrm{Ca}, \mathrm{Sr})_{3} \mathrm{Ti}_{2} \mathrm{O}_{7}$ crystals. Nature Materials 14 (4), 407-413.

54. Pitcher, M.J., et al., 2015. Tilt engineering of spontaneous polarization and magnetization above $300 \mathrm{~K}$ in a bulk layered perovskite. Science 347 (6220), 420-424.

55. Efremov, D.V., Van den Brink, J., Khomskii, D.I., 2004. Bond-versus site-centred ordering and possible ferroelectricity in manganites. Nature Materials 3 (12), $853-856$.

56. Giovannetti, G., Kumar, S., van den Brink, J., Picozzi, S., 2009. Magnetically induced electronic ferroelectricity in half-doped manganites. Physical Review Letters 103 (3), 037601.

57. Lopes, A.M.L., et al., 2008. New phase transition in the $\mathrm{Pr}_{1-x} \mathrm{Ca}_{x} \mathrm{MnO}_{3}$ system: Evidence for electrical polarization in charge ordered manganites. Physical Review Letters $100(15), 155702$.

58. Ikeda, N., et al., 2005. Ferroelectricity from iron valence ordering in the charge-frustrated system LuFe $0_{4}$. Nature 436 (7054), 1136-1138.

59. de Groot, J., et al., 2012. Charge order in $\mathrm{LuFe}_{2} \mathrm{O}_{4}$ : An unlikely route to ferroelectricity. Physical Review Letters 108 (18), 187601.

60. Lafuerza, S., et al., 2014. Determination of the sequence and magnitude of charge order in LuFe $\mathrm{O}_{4}$ by resonant X-ray scattering. Physical Review B 90 (8), 085130.

61. Yamauchi, K., Barone, P., 2014. Electronic ferroelectricity induced by charge and orbital orderings. Journal of Physics-Condensed Matter 26 (10), 103201.

62. van den Brink, J., Khomskii, D.I., 2008. Multiferroicity due to charge ordering. Journal of Physics-Condensed Matter 20 (43), 434217.

63. Tokura, Y., Seki, S., Nagaosa, N., 2014. Multiferroics of spin origin. Reports on Progress in Physics 77 (7), 076501.

64. Goto, T., Kimura, T., Lawes, G., Ramirez, A.P., Tokura, Y., 2004. Ferroelectricity and giant magnetocapacitance in perovskite rare-earth manganites. Physical Review Letters 92 (25), 257201.

65. Kenzelmann, M., et al., 2005. Magnetic inversion symmetry breaking and ferroelectricity in $\mathrm{TbMnO}_{3}$. Physical Review Letters 95 (8), 087206.

66. Mostovoy, M., 2006. Ferroelectricity in spiral magnets. Physical Review Letters 96 (6), 067601.

67. Dzyaloshinskii, I., 1964. Theory of helical structures in antiferromagnets I: Nonmetals. Soviet Physics JETP 19, 960-971.

68. Moriya, T., 1960. Anisotropic superexchange interaction and weak ferromagnetism. Physical Review 120 (1), 91-98.

69. Sergienko, I.A., Dagotto, E., 2006. Role of the Dzyaloshinskii-Moriya interaction in multiferroic perovskites. Physical Review B 73 (9), 094434.

70. Walker, H.C., et al., 2011. Femtoscale magnetically induced lattice distortions in multiferroic $\mathrm{TbMnO}_{3}$. Science 333 (6047), 1273-1276.

71. Yamasaki, Y., et al., 2007. Electric control of spin helicity in a magnetic ferroelectric. Physical Review Letters 98 (14), 147204.

72. Katsura, H., Nagaosa, N., Balatsky, A.V., 2005. Spin current and magnetoelectric effect in noncollinear magnets. Physical Review Letters 95 (5), 057205

73. Mochizuki, M., Furukawa, N., Nagaosa, N., 2011. Theory of spin-phonon coupling in multiferroic manganese perovskites RMnO3. Physical Review B 84 (14), 144409.

74. Arima, T., et al., 2006. Collinear to spiral spin transformation without changing the modulation wavelength upon ferroelectric transition in $\mathrm{Tb}_{1-x} \mathrm{Dy}_{\mathrm{x}} \mathrm{MnO}_{3}$. $\mathrm{Physical} \mathrm{Review}$ Letters 96 (9), 097202

75. Mochizuki, M., Furukawa, N., 2009. Microscopic model and phase diagrams of the multiferroic perovskite manganites. Physical Review B 80 (13), 134416.

76. Kimura, T., Sekio, Y., Nakamura, H., Siegrist, T., Ramirez, A.P., 2008. Cupric oxide as an induced-multiferroic with high-Tc. Nature Materials 7 (4), 291-294.

77. Kitagawa, Y., et al., 2010. Low-field magnetoelectric effect at room temperature. Nature Materials 9 (10), 797-802.

78. Jia, C., Onoda, S., Nagaosa, N., Han, J.H., 2006. Bond electronic polarization induced by spin. Physical Review B 74 (22), 224444. 
79. Jia, C., Onoda, S., Nagaosa, N., Han, J.H., 2007. Microscopic theory of spin-polarization coupling in multiferroic transition metal oxides. Physical Review B 76 (14), 144424.

80. Zheludev, A., Sato, T., Masuda, T., Uchinokura, K., Shirane, G., 2003. Spin waves and the origin of commensurate magnetism in $\mathrm{Ba}_{2} \mathrm{CoGe}_{2} \mathrm{O}_{7}$. Physical Review B 68 (2), 024428.

81. Sato, T., Masuda, T., Uchinokura, K., 2003. Magnetic property of $\mathrm{Ba}_{2} \mathrm{CoGe}_{2} \mathrm{O}_{7}$. Physica B-Condensed Matter 329, 880-881.

82. Yi, H.T., Choi, Y.J., Lee, S., Cheong, S.W., 2008. Multiferroicity in the square-lattice antiferromagnet of $\mathrm{Ba}_{2} \mathrm{CoGe}_{2} \mathrm{O}_{7}$. Applied Physics Letters 92 (21), 212904.

83. Murakawa, H., Onose, Y., Miyahara, S., Furukawa, N., Tokura, Y., 2010. Ferroelectricity induced by spin-dependent metal-ligand hybridization in $\mathrm{Ba}_{2} \mathrm{CoGe}_{2} \mathrm{O}_{7}$. Physical Review Letters 105 (13), 137202

84. Yamauchi, K., Barone, P., Picozzi, S., 2011. Theoretical investigation of magnetoelectric effects in $\mathrm{Ba}_{2} \mathrm{CoGe}_{2} \mathrm{O}_{7}$. Physical Review B 84 (16), 165137.

85. Perez-Mato, J.M., Ribeiro, J.L., 2011. On the symmetry and the signature of atomic mechanisms in multiferroics: The example of $\mathrm{Ba}_{2} \mathrm{CoGe}_{2} \mathrm{O}_{7}$. Acta $\mathrm{Crystallographica}$ Section A 67, 264-268.

86. Toledano, P., Khalyavin, D.D., Chapon, L.C., 2011. Spontaneous toroidal moment and field-induced magnetotoroidic effects in $\mathrm{Ba}_{2} \mathrm{CoGe}_{2} \mathrm{O}_{7}$. Physical Review B 84 (9), 094421.

87. Mitamura, H., et al., 2007. Dielectric polarization measurements on the antiferromagnetic triangular lattice system $\mathrm{CuFeO}_{2}$ in pulsed high magnetic fields. Journal of the Physical Society of Japan 76 (9), 094709.

88. Mekata, M., Yaguchi, N., Takagi, T., Mitsuda, S., Yoshizawa, H., 1992. Magnetic ordering in delaffossite $\mathrm{CuFeO}_{2}$. Journal of Magnetism and Magnetic Materials 104 , 823-824.

89. Kimura, T., Lashley, J.C., Ramirez, A.P., 2006. Inversion-symmetry breaking in the noncollinear magnetic phase of the triangular-lattice antiferromagnet $\mathrm{CuFeO}_{2}$. Physical Review B 73 (22), 220401.

90. Nakajima, T., et al., 2007. Spin noncollinearlity in multiferroic phase of triangular lattice antiferromagnet $\mathrm{CuFe}_{1-x} \mathrm{Al}_{x} \mathrm{O}_{2}$. Journal of the Physical Society of Japan 76 (4), 043709.

91. Seki, S., et al., 2007. Impurity-doping-induced ferroelectricity in the frustrated antiferromagnet $\mathrm{CuFeO}_{2}$. Physical Review B 75 (10), 100403.

92. Terada, N., 2014. Spin and orbital orderings behind multiferroicity in delafossite and related compounds. Journal of Physics-Condensed Matter 26 (45), 453202.

93. Arima, T.H., 2007. Ferroelectricity induced by proper-screw type magnetic order. Journal of the Physical Society of Japan 76 (7), 073702.

94. Kaplan, T.A., Mahanti, S.D., 2011. Canted-spin-caused electric dipoles: A local symmetry theory. Physical Review B 83 (17), 174432.

95. Tanaka, Y., et al., 2012. Incommensurate orbital modulation behind ferroelectricity in $\mathrm{CuFeO}_{2}$. Physical Review Letters 109 (12), 127205.

96. Perks, N.J., Johnson, R.D., Martin, C., Chapon, L.C., Radaelli, P.G., 2012. Magneto-orbital helices as a route to coupling magnetism and ferroelectricity in multiferroic $\mathrm{CaMn}_{7} \mathrm{O}_{12}$. Nature Communications 3,6 .

97. Zhang, G.Q., et al., 2011. Multiferroic properties of $\mathrm{CaMn}_{7} \mathrm{O}_{12}$. Physical Review B 84 (17), 174413.

98. Johnson, R.D., et al., 2012. Giant improper ferroelectricity in the ferroaxial magnet $\mathrm{CaMn}_{7} 0_{12}$. Physical Review Letters 108 (6), 067201.

99. Goodenough, J.B., 1964. Magnetism and the Chemical Bond. New York: Wiley Interscience.

100. Simonet, V., Loire, M., Ballou, R., 2012. Magnetic chirality as probed by neutron scattering. European Physical Journal-Special Topics 213 (1). 5-36.

101. Cao, K., Johnson, R.D., Perks, N., Giustino, F., Radaelli, P.G., 2015. First-principles study of structurally modulated multiferroic CaMn $70_{12}$. Physical Review B 91 (6), 064422.

102. Chapon, L.C., Radaelli, P.G., Blake, G.R., Park, S., Cheong, S.W., 2006. Ferroelectricity induced by acentric spin-density waves in $\mathrm{YMn}_{2} \mathrm{O}_{5}$. Physical Review Letters 96 (9), 097601.

103. Choi, Y.J., et al., 2008. Ferroelectricity in an ising chain magnet. Physical Review Letters 100 (4), 047601.

104. Zubkov, V.G., Bazuev, G.G., Tyutyunnik, A.P., Berger, I.F., 2001. Synthesis, crystal structure, and magnetic properties of quasi-one-dimensional oxides $\mathrm{Ca}_{3} \mathrm{CuMnO} \mathrm{O}_{6}$ and $\mathrm{Ca}_{3} \mathrm{C}_{1}+x \mathrm{Mn}_{1-x} \mathrm{O}_{6}$. Journal of Solid State Chemistry 160 (2), 293-301.

105. Wu, H., et al., 2009. Ising magnetism and ferroelectricity in $\mathrm{Ca}_{3} \mathrm{CoMnO}_{6}$. Physical Review Letters 102 (2), 026404

106. Kim, J.H., et al., 2008. Spiral spin structures and origin of the magnetoelectric coupling in $\mathrm{YMn}_{2} \mathrm{O}_{5}$. Physical Review B 78 (24), 245115.

107. Wakimoto, S., et al., 2013. Role of magnetic chirality in polarization flip upon a commensurate-incommensurate magnetic phase transition in $\mathrm{YMn}_{2} \mathrm{O}_{5}$. Physical Review $\mathrm{B}$ 88 (14), 140403

108. Chaudhury, R.P., et al., 2008. Pressure-induced polarization reversal in multiferroic $\mathrm{YMn}_{2} \mathrm{O}_{5}$. Physical Review B 77 (22), 220104.

109. Kimura, H., Noda, Y., Kohn, K., 2009. Spin-driven ferroelectricity in the multiferroic compounds of $\mathrm{RMn}_{2} \mathrm{O}_{5}$. Journal of Magnetism and Magnetic Materials 321 (7), 854-857.

110. Ishiwata, S., Tokunaga, Y., Taguchi, Y., Tokura, Y., 2011. High-pressure hydrothermal crystal growth and multiferroic properties of a perovskite $\mathrm{YMnO}_{3}$. Journal of the American Chemical Society 133 (35), 13818-13820.

111. Sergienko, I.A., Sen, C., Dagotto, E., 2006. Ferroelectricity in the magnetic e-phase of orthorhombic perovskites. Physical Review Letters 97 (22), 227204.

112. Picozzi, S., et al., 2008. Microscopic mechanisms for improper ferroelectricity in multiferroic perovskites: A theoretical review. Journal of Physics-Condensed Matter $20(43), 434208$

113. Lorenz, B., Wang, Y.Q., Sun, Y.Y., Chu, C.W., 2004. Large magnetodielectric effects in orthorhombic $\mathrm{HoMnO}_{3}$ and $\mathrm{YMnO}_{3}$. Physical Review B 70 (21), 212412.

114. Lorenz, B., Wang, Y.Q., Chu, C.W., 2007. Ferroelectricity in perovskite $\mathrm{HoMnO}_{3}$ and $\mathrm{YMnO}_{3}$. Physical Review B 76 (10), 104405.

115. Kimura, T., Lawes, G., Goto, T., Tokura, Y., Ramirez, A.P., 2005. Magnetoelectric phase diagrams of orthorhombic $\mathrm{RMnO}_{3}(\mathrm{R}=\mathrm{Gd}$, Tb, and Dy). Physical Review B 71 (22), 224425.

116. Lu, C.L., Dong, S., Wang, K.F., Liu, J.M., 2010. Enhanced polarization and magnetoelectric response in $\mathrm{Tb}_{1-x} \mathrm{Ho}_{x} \mathrm{MnO}_{3}$. Applied Physics A-Materials Science \& Processing 99 (1), 323-331.

117. Pomjakushin, V.Y., et al., 2009. Evidence for large electric polarization from collinear magnetism in $\mathrm{TmMnO}_{3}$. New Journal of Physics 11,043019

118. Ishiwata, S., et al., 2010. Perovskite manganites hosting versatile multiferroic phases with symmetric and antisymmetric exchange strictions. Physical Review B 81 (10), 100411.

119. Fontcuberta, J., 2015. Multiferroic $\mathrm{RMnO}_{3}$ thin films. Comptes Rendus Physique 16 (2), 204-226.

120. Sando, D., Barthelemy, A., Bibes, M., 2014. $\mathrm{BiFeO}_{3}$ epitaxial thin films and devices: Past, present and future. Journal of Physics-Condensed Matter 26 (47), 473201.

121. Ramesh, R., Spaldin, N.A., 2007. Multiferroics: Progress and prospects in thin films. Nature Materials 6 (1), 21-29.

122. Martin, L., et al., 2008. Multiferroics and magnetoelectrics: Thin films and nanostructures. Journal of Physics-Condensed Matter 20 (43), 434220.

123. Lu, C., Hu, W.J., Tian, Y.F., Wu, T., 2015. Multiferroic oxide thin films and heterostructures. Applied Physics Reviews 2 (2), 021304.

124. Ma, J., Hu, J.M., Li, Z., Nan, C.W., 2011. Recent progress in multiferroic magnetoelectric composites: From bulk to thin films. Advanced Materials 23 (9), $1062-1087$.

125. Velev, J.P., Jaswal, S.S., Tsymbal, E.Y., 2011. Multi-ferroic and magnetoelectric materials and interfaces. Philosophical Transactions of the Royal Society a-Mathematical Physical and Engineering Sciences 369 (1948), 3069-3097.

126. Zavaliche, F., et al., 2005. Electric field-induced magnetization switching in epitaxial columnar nanostructures. Nano Letters 5 (9), 1793-1796.

127. Zheng, $\mathrm{H}$., et al., 2004. Multiferroic $\mathrm{BaTiO}_{3}-\mathrm{CoFe}_{2} \mathrm{O}_{4}$ nanostructures. Science 303 (5658), 661-663.

128. Garcia, V., Bibes, M., Barthelemy, A., 2015. Artificial multiferroic heterostructures for an electric control of magnetic properties. Comptes Rendus Physique 16 (2), 168-181. 
129. Liu, M., Sun, N.X., 2014. Voltage control of magnetism in multiferroic heterostructures. Philosophical Transactions of the Royal Society a-Mathematical Physical and Engineering Sciences 372 (2009), 20120439.

130. Vaz, C.A.F., 2012. Electric field control of magnetism in multiferroic heterostructures. Journal of Physics-Condensed Matter 24 (33), 333201.

131. He, X., et al., 2010. Robust isothermal electric control of exchange bias at room temperature. Nature Materials 9 (7), 579-585.

132. Rondinelli, J.M., Stengel, M., Spaldin, N.A., 2008. Carrier-mediated magnetoelectricity in complex oxide heterostructures. Nature Nanotechnology 3 (1), 46-50.

133. Zhang, S.F., 1999. Spin-dependent surface screening in ferromagnets and magnetic tunnel junctions. Physical Review Letters 83 (3), 640-643.

134. Seidel, J., et al., 2009. Conduction at domain walls in oxide multiferroics. Nature Materials 8 (3), 229-234.

135. Geng, Y.N., et al., 2012. Collective magnetism at multiferroic vortex domain walls. Nano Letters 12 (12), 6055-6059.

136. Choi, T., et al., 2010. Insulating interlocked ferroelectric and structural antiphase domain walls in multiferroic $\mathrm{YMnO}_{3}$. Nature Materials 9 (3), $253-258$.

137. Catalan, G., Seidel, J., Ramesh, R., Scott, J.F., 2012. Domain wall nanoelectronics. Reviews of Modern Physics 84 (1), 119-156.

138. Matzen, S., Fusil, S., 2015. Domains and domain walls in multiferroics. Comptes Rendus Physique 16 (2), 227-240.

139. Catalan, G., 2014. Physics of ferroic and multiferroic domain walls. In: Saxena, A., Planes, A. (Eds.), Mesoscopic Phenomena in Multifunctional Materials. Berlin Heidelberg: Springer, pp. 225-247. 